\title{
Rhodium-Catalyzed Ring-Opening Reactions of $N$-Boc-Azabenzonorbornadienes with Amine Nucleophiles
}

Yong-Hwan Cho, Valentin Zunic, Hisanori Senboku, Madeline Olsen and

Mark Lautens*

Davenport Laboratories, Department of Chemistry, University of Toronto

80 St. George Street, Toronto, Ontario, Canada M5H 3 H6

\section{Supporting Information}

General. All moisture and air sensitive manipulations were carried out under a dried nitrogen atmosphere. NMR spectra were recorded on Varian Mercury NMR spectrometer $\left(300 \mathrm{MHz}\right.$ for ${ }^{1} \mathrm{H}, 75 \mathrm{MHZ}$ for ${ }^{13} \mathrm{C}, 121 \mathrm{MHz}$ for ${ }^{31} \mathrm{P}$, and $282 \mathrm{MHz}$ for ${ }^{19} \mathrm{~F}$ ). Chemical shifts are reported in $\delta$ ppm referenced to $\mathrm{SiMe}_{4}$ standard for ${ }^{1} \mathrm{H} \mathrm{NMR}$, chloroform- $d(\delta 77.0)$ for ${ }^{13} \mathrm{C}$ NMR, an external $85 \% \mathrm{H}_{3} \mathrm{PO}_{4}$ standard for ${ }^{31} \mathrm{P} \mathrm{NMR}$ and an external $\mathrm{CFCl}_{3}$ standard for ${ }^{19} \mathrm{~F}$ NMR. IR spectra were obtained using a Perkin-Elmer Spectrum 1000 FT-IR spectrometer as a neat film on a $\mathrm{NaCl}$ plate. High resolution mass spectra were obtained from a Micromass 70S-250 mass spectrometer (EI) or an ABI/Sciex Qstar mass spectrometer (ESI). Optical rotations were measured on a Perkin-Elmer Model 243 Polarimeter and melting points were taken on a Fisher-Johns melting point apparatus. HPLC analysis was performed on an Agilent 1100 Series HPLC with Chiralcel OD, OD-H, AD or Chiralpak AS columns.

Materials. Dioxane, $\mathrm{Et}_{2} \mathrm{O}, \mathrm{THF}, \mathrm{THP}$, and toluene were distilled from sodium benzophenone ketyl immediately prior to use. $\mathrm{CH}_{2} \mathrm{Cl}_{2}$ and $\mathrm{CH}_{3} \mathrm{CN}$ were distilled from calcium hydride. DMF was dried over $\mathrm{MgSO}_{4}$ and stored over activated molecular sieves. Azabenzonorbornadienes $\mathbf{1 f},{ }^{1} \mathbf{1 g},{ }^{2} \mathbf{1 h},{ }^{3} \mathbf{1 l},{ }^{4} \mathbf{1 m},{ }^{4}$ and $\mathbf{4 a}^{5}$ were prepared according to the reported procedure.

\section{Preparation of 1,4-Dihydro-1,4-epiazano-naphthalene-9-carbamic acid tert-butyl} ester (1a): To a stirred solution of anthranilic acid $(8.0 \mathrm{~g}, 58.3 \mathrm{mmol})$ and trichloroacetic acid $(67 \mathrm{mg}, 0.4 \mathrm{mmol})$ in dry THF $(120 \mathrm{~mL})$ was slowly added isoamyl nitrite $(13 \mathrm{~mL}$, $93.3 \mathrm{mmol}$ ) at $0{ }^{\circ} \mathrm{C}$ for $30 \mathrm{~min}$ and the mixture was stirred at room temperature for $1.5 \mathrm{~h}$. The dark yellow precipitate of benzenediazonium-2-carboxylate was collected by filtration and washed with cold THF using minimal suction for draining so as to prevent 
complete drying. The salt was immediately transferred to a flask and 1,2-dichloroethane $(100 \mathrm{~mL})$ and $N$-tert-butyl 1-pyrrolecarboxylate $(7.8 \mathrm{~mL}, 46.7 \mathrm{mmol})$ were added. The mixture was heated at $60{ }^{\circ} \mathrm{C}$ for $45 \mathrm{~min}$ and the black mixture was cooled to room temperature. After concentration under reduced pressure, the residue was purified by flash column chromatography (hexane/ethyl acetate $=95 / 5$ ) and recrystallization with hexane to give $7.8 \mathrm{~g}$ of $\mathbf{1 a}\left(55 \%\right.$ yield). mp $70-72{ }^{\circ} \mathrm{C}$ (lit. ${ }^{6} \mathrm{mp} 72-73{ }^{\circ} \mathrm{C}$ for $\left.1 \mathrm{a}\right)$; IR $\left(\mathrm{NaCl}, \mathrm{cm}^{-1}\right)$ 2976, 2931, 1708, 1455, 1367, 1332, 1252, 1167, 1074, 857, 830, 782, 748, 694, 643. ${ }^{1} \mathrm{H}$ NMR $\left(\mathrm{CDCl}_{3}\right) \delta 1.37$ (s, 9H), 5.48 (bs, 2H), 6.94-6.98 (m, 4H), 7.22-7.29 $(\mathrm{m}, 2 \mathrm{H}) ;{ }^{13} \mathrm{C} \mathrm{NMR}\left(\mathrm{CDCl}_{3}\right) \delta 155.3,148.5,143.1(\mathrm{~d}, J=85.0 \mathrm{~Hz}), 125.1,121.0(\mathrm{~d}, J=$ $31.5 \mathrm{~Hz}), 80.7,66.7$ (d, $J=47.3 \mathrm{~Hz}), 28.3$.

\section{Preparation of 1,4-Dihydro-1,4-epiazano-naphthalene-9-carbamic acid methyl} ester (1b): To a solution of 1,4-dihydro-1,4-epiazano-naphthalene ${ }^{7}(0.5 \mathrm{~g}, 3.5 \mathrm{mmol})$ and triethylamine $(2.5 \mathrm{~mL})$ in dry $\mathrm{CH}_{2} \mathrm{Cl}_{2}(10 \mathrm{~mL})$ was added methyl chloroformate $(0.8 \mathrm{~mL}, 10.5 \mathrm{mmol})$ dropwise at $0{ }^{\circ} \mathrm{C}$ over $10 \mathrm{~min}$ and the reaction was stirred at room temperature for $12 \mathrm{~h}$. The mixture was quenched with saturated $\mathrm{NaHCO}_{3}$ solution and extracted with $\mathrm{CH}_{2} \mathrm{Cl}_{2}$. The organic layer was dried over anhydrous $\mathrm{Na}_{2} \mathrm{SO}_{4}$ and concentrated under reduced pressure. The residue was purified by flash column chromatography (hexane/ethyl acetate $=90 / 10)$ to give $0.6 \mathrm{~g}$ of $\mathbf{1 b}(85 \%$ yield). $\mathrm{mp}$ 48-50 ${ }^{\circ} \mathrm{C}$; IR $\left(\mathrm{NaCl}, \mathrm{cm}^{-1}\right)$ 3027, 2953, 1717, 1441, 1333, 1250, 1190, 1091, 952, 856, 813, 742, 695. ${ }^{1} \mathrm{H}$ NMR $\left(\mathrm{CDCl}_{3}\right) \delta 3.61(\mathrm{~s}, 3 \mathrm{H}), 5.54(\mathrm{~s}, 2 \mathrm{H}), 6.93-6.96(\mathrm{~m}, 4 \mathrm{H}), 7.23$ (bs, $2 \mathrm{H}) ;{ }^{13} \mathrm{C} \mathrm{NMR}\left(\mathrm{CDCl}_{3}\right) \delta 155.7,148.1,143.1(\mathrm{~d}, J=86.5 \mathrm{~Hz}), 125.1,120.1(\mathrm{~d}, J=$ $25.0 \mathrm{~Hz}), 66.2,52.7$. HRMS calcd for $\mathrm{C}_{12} \mathrm{H}_{11} \mathrm{NO}_{2}\left(\mathrm{M}^{+}\right)$: 201.0789. Found: 201.0791. The azabenzonorbornadienes $\mathbf{1 c}, \mathbf{1 d}$, and, $1 \mathbf{e}$ were prepared in a similar manner.

1,4-Dihydro-1,4-epiazano-naphthalene-9-carbamic acid benzyl ester (1c): $80 \%$ yield. $\mathrm{mp} 85-87{ }^{\circ} \mathrm{C}$; IR $\left(\mathrm{NaCl}, \mathrm{cm}^{-1}\right) 3031,1710,1454,1387,1326,1244,1071,805$, 748, 694. ${ }^{1} \mathrm{H}$ NMR $\left(\mathrm{CDCl}_{3}\right) \delta 5.05$ (s, 2H), 5.57 (s, 2H), 6.92-6.96 (m, 4H), 7.20-7.32 $(\mathrm{m}, 7 \mathrm{H}) ;{ }^{13} \mathrm{C} \mathrm{NMR}\left(\mathrm{CDCl}_{3}\right) \delta 155.3,148.1,143.1(\mathrm{~d}, J=93.3 \mathrm{~Hz}), 136.3,128.5,128.1$, 127.8, 125.2, $121.1(\mathrm{~d}, J=35.7 \mathrm{~Hz}), 67.2$, 66.3. HRMS calcd for $\mathrm{C}_{18} \mathrm{H}_{15} \mathrm{NO}_{2}\left(\mathrm{M}^{+}\right)$: 277.1102. Found: 277.1102 .

(1,4-Dihydro-1,4-epiazano-naphthalen-9-yl)-benzamide (1d): 90\% yield. mp 94-97 ${ }^{\circ} \mathrm{C}$; IR $\left(\mathrm{NaCl}, \mathrm{cm}^{-1}\right) 3058,1714,1647,1448,1370,1258,1071,784 .{ }^{1} \mathrm{H}$ NMR $\left(\mathrm{CDCl}_{3}\right)$ $\delta 5.52(\mathrm{~s}, 1 \mathrm{H}), 6.00(\mathrm{~s}, 1 \mathrm{H}), 6.88-6.89(\mathrm{~m}, 1 \mathrm{H}), 6.94-7.00(\mathrm{~m}, 2 \mathrm{H}), 7.16-7.17(\mathrm{~m}, 2 \mathrm{H})$, 7.34-7.54 (m, 6H); ${ }^{13} \mathrm{C} \mathrm{NMR}\left(\mathrm{CDCl}_{3}\right) \delta 167.5,143.5(\mathrm{~d}, J=23.8 \mathrm{~Hz}), 134.5,131.0$, 
128.5, 127.9, 125.3 (d, $J=25.8 \mathrm{~Hz}), 121.6,120.4,66.1$ (d, $J=43.2 \mathrm{~Hz})$. HRMS calcd for $\mathrm{C}_{17} \mathrm{H}_{13} \mathrm{NO}(\mathrm{M}+)$ : 247.0997. Found: 247.0997.

1-(1,4-Dihydro-1,4-epiazano-naphthalen-9-yl)-acetamide (1e): $85 \%$ yield, $\mathrm{mp}$ 63-64 ${ }^{\circ} \mathrm{C}$; IR $\left(\mathrm{NaCl}, \mathrm{cm}^{-1}\right) 3072,1654,1444,1394,1337,1247,1032,795,752 .{ }^{1} \mathrm{H}$ $\operatorname{NMR}\left(\mathrm{CDCl}_{3}\right) \delta 1.95(\mathrm{~s}, 3 \mathrm{H}), 5.50(\mathrm{~s}, 1 \mathrm{H}), 5.87(\mathrm{~s}, 1 \mathrm{H}), 6.95-6.98(\mathrm{~m}, 3 \mathrm{H}), 7.05(\mathrm{dd}, J$ $=5.6 \mathrm{~Hz}, 2.4 \mathrm{~Hz}, 1 \mathrm{H}), 7.24-7.29(\mathrm{~m}, 2 \mathrm{H}) ;{ }^{13} \mathrm{C} \mathrm{NMR}\left(\mathrm{CDCl}_{3}\right) \delta 166.8,148.4,148.0$, $144.3,142.3,125.3,125.0,121.4,120.3,66.2,63.3,21.4$. HRMS calcd for $\mathrm{C}_{12} \mathrm{H}_{11} \mathrm{NO}$ $\left(\mathrm{M}^{+}\right)$: 185.0840. Found: 185.0833 .

Preparation of 1,4-dihydro-1,4-epiazano-5,8-dimethylnaphthalene-9-carbamic acid tert-butyl ester (1i): To a stirred solution of 3.6-dimethylanthranilic acid ${ }^{8}(14.0 \mathrm{~g}$, $84.7 \mathrm{mmol})$ and trichloroacetic acid $(0.2 \mathrm{~g}, 0.9 \mathrm{mmol})$ in dry THF $(100 \mathrm{~mL})$ was slowly added isoamyl nitrite $(14 \mathrm{~mL}, 101.7 \mathrm{mmol})$ at $-20{ }^{\circ} \mathrm{C}$ for $40 \mathrm{~min}$ by syringe pump under nitrogen atmosphere. The mixture was allowed to stir at $-20{ }^{\circ} \mathrm{C}$ for another $2 \mathrm{~h}$. Then, $N$-tert-butyl 1-pyrrolecarboxylate $(11 \mathrm{~mL}, 67.8 \mathrm{mmol})$ and 1,2-dichloroethane $(100 \mathrm{~mL})$ was added and the mixture was heated immediately at $80{ }^{\circ} \mathrm{C}$ for $30 \mathrm{~min}$. The resulting mixture was then cooled to room temperature and all volatile substrates were removed under reduced pressure. The residue was purified by flash column chromatography (hexane/ethyl acetate $=95 / 5)$ and recrystallization with hexane to give $6.9 \mathrm{~g}(30 \%$ yield $)$ of 1i. mp 95-97 ${ }^{\circ} \mathrm{C}$; IR $\left(\mathrm{NaCl}, \mathrm{cm}^{-1}\right) 2974,1709,1490,1455,1366,1329,1253,1167$, 1073. ${ }^{1} \mathrm{H}$ NMR $\left(\mathrm{CDCl}_{3}\right) \delta 1.38(\mathrm{~s}, 9 \mathrm{H}), 2.29(\mathrm{~s}, 6 \mathrm{H}), 5.53-5.57(\mathrm{~m}, 2 \mathrm{H}), 6.67(\mathrm{~s}, 2 \mathrm{H})$, 6.95-6.99 (m, 2H); ${ }^{13} \mathrm{C} \mathrm{NMR}\left(\mathrm{CDCl}_{3}\right) \delta 155.5,146.3,142.9(\mathrm{~d}, J=97.9 \mathrm{~Hz}), 127.9(\mathrm{~d}, J$ $=38.5 \mathrm{~Hz}), 126.6,80.6,65.2(\mathrm{~d}, J=42.9 \mathrm{~Hz}), 28.3,17.9$. Anal. Calcd for $\mathrm{C}_{17} \mathrm{H}_{21} \mathrm{NO}_{2}: \mathrm{C}$, 75.25; H, 7.80; N, 5.16. Found: C, 75.19; H, 7.71; N, 5.19.

\section{Preparation of 1,4-dihydro-1,4-epiazano-5,6,7,8-tetramethyl-naphthalene-9-} carbamic acid tert-butyl ester (1j): To a solution of 1,2-dibromotetramethylbenzene 9 (20.0 g, $68.5 \mathrm{mmol}$ ) prepared from commercially available tetramethylbenzene according to previously reported procedure ${ }^{10}$ in dry toluene $(200 \mathrm{~mL})$ was slowly added $n$-butyllithium solution in hexane $(95 \mathrm{~mL}, 150.7 \mathrm{mmol})$ diluted with dry toluene (24 $\mathrm{mL})$ and $N$-tert-butyl 1-pyrrolecarboxylate solution in dry toluene $(14 \mathrm{~mL}, 82.2 \mathrm{mmol}$ in $110 \mathrm{~mL}$ of toluene) at $0{ }^{\circ} \mathrm{C}$ by syringe pump for $3 \mathrm{~h}$ under nitrogen atmosphere. The mixture was allowed to stir at room temperature for $48 \mathrm{~h}$ and was quenched with water. The resulting mixture was extracted with ethyl acetate and the organic phase was dried over anhydrous $\mathrm{MgSO}_{4}$. After removal of all volatile solvents under reduced pressure, flash column chromatography on silica gel (hexane/ethyl acetate $=95 / 5$ ) and 
recrystallization with hexane gave $7.0 \mathrm{~g}$ (34\% yield) of $\mathbf{1 j}$. mp $125-127{ }^{\circ} \mathrm{C}$; IR $(\mathrm{NaCl}$, $\left.\mathrm{cm}^{-1}\right) 2973,1708,1366,1330,1257,1167,1073 .{ }^{1} \mathrm{H} \mathrm{NMR}\left(\mathrm{CDCl}_{3}\right) \delta 1.39(\mathrm{~s}, 9 \mathrm{H}), 2.11$ $(\mathrm{s}, 6 \mathrm{H}), 2.24(\mathrm{~s}, 6 \mathrm{H}), 5.56-5.60(\mathrm{~m}, 2 \mathrm{H}), 6.94-6.98(\mathrm{~m}, 2 \mathrm{H}) ;{ }^{13} \mathrm{C} \mathrm{NMR}\left(\mathrm{CDCl}_{3}\right) \delta 155.3$, 143.5, 143.1 (d, $J=101.6 \mathrm{~Hz}), 131.6,127.2(\mathrm{~d}, J=40.1 \mathrm{~Hz}), 80.5,65.8$ (d, $J=46.1 \mathrm{~Hz})$, 28.4, 16.5, 16.0. Anal. Calcd for $\mathrm{C}_{19} \mathrm{H}_{25} \mathrm{NO}_{2}$ : C, 76.32; H, 8.42; N, 4.68. Found: $\mathrm{C}$, $76.71 ; \mathrm{H}, 8.59 ; \mathrm{N}, 4.65$.

\section{1,4-dihydro-1,4-epiazano-6,7-dimethyl-naphthalene-9-carbamic acid tert-butyl} ester (1k): Prepared from 1,2-dibromo-4,5-dimethylbenzene ${ }^{11}$ in a similar manner to $\mathbf{1 j}$ in $35 \%$ yield. $\mathrm{mp} 93-95^{\circ} \mathrm{C}$; IR $\left(\mathrm{NaCl}, \mathrm{cm}^{-1}\right) 2974,1706,1593,1455,1366,1328,1251$, 1167, 1071. ${ }^{1} \mathrm{H}$ NMR $\left(\mathrm{CDCl}_{3}\right) \delta 1.38(\mathrm{~s}, 9 \mathrm{H}), 2.17(\mathrm{~s}, 6 \mathrm{H}), 5.42(\mathrm{~m}, 2 \mathrm{H}), 6.95(\mathrm{~m}, 2 \mathrm{H})$, $7.05(\mathrm{~s}, 2 \mathrm{H}) ;{ }^{13} \mathrm{C}$ NMR $\left(\mathrm{CDCl}_{3}\right) \delta 155.2,146.0,143.3(\mathrm{~d}, J=81.2 \mathrm{~Hz}), 132.6,122.9(\mathrm{~d}$, $J=27.1 \mathrm{~Hz}), 80.6,66.4(\mathrm{~d}, J=47.5 \mathrm{~Hz}), 28.4$, 19.9. Anal. Calcd for $\mathrm{C}_{17} \mathrm{H}_{21} \mathrm{NO}_{2}: \mathrm{C}$, 75.25; H, 7.80; N, 5.16. Found: C, 75.10; H, 7.89; N, 5.07.

\section{Rhodium-Catalyzed Asymmetric Ring-Opening of 1a with Pyrrolidine (2a) in the} presence of triethylamine hydrochloride in tetrahydropyran. The reaction conditions and results are summarized in Tables 2 and 6. A typical procedure is given for the reaction of $\mathbf{1 a}$ with 5.0 eq. of pyrrolidine (2a) in the presence of $2.5 \mathrm{~mol} \%$ of $[\mathrm{Rh}(\mathrm{cod}) \mathrm{Cl}]_{2}$ and $11.0 \mathrm{~mol} \%$ of $\left(S, S^{\prime}\right)-\left(R, R^{\prime}\right)-\mathrm{C}_{2}$-Ferriphos (4a) (entry 14 in Table 2$)$.

To a stirred solution of $1 \mathrm{a}(80 \mathrm{mg}, 0.33 \mathrm{mmol})$, triethylamine hydrochloride (45 $\mathrm{mg}$, $0.33 \mathrm{mmol}),[\mathrm{Rh}(\mathrm{cod}) \mathrm{Cl}]_{2}(4 \mathrm{mg}, 8 \mu \mathrm{mol})$, and $4 \mathbf{a}(26 \mathrm{mg}, 36 \mu \mathrm{mol})$ in dry tetrahydropyran $(1.6 \mathrm{~mL})$ was added pyrrolidine $(0.14 \mathrm{~mL}, 1.6 \mathrm{mmol})$ and the mixture was allowed to stir at $80{ }^{\circ} \mathrm{C}$ for $72 \mathrm{~h}$ under nitrogen atmosphere. The resulting mixture was quenched with $1 \mathrm{~N} \mathrm{NaOH}$ solution and was extracted with $\mathrm{CH}_{2} \mathrm{Cl}_{2}$. The separated organic layer was dried over anhydrous $\mathrm{MgSO}_{4}$ and was concentrated under reduced pressure. The residue was purified by flash column chromatography (hexane/ethyl acetate $=70 / 30)$ to give $80 \mathrm{mg}(78 \%$ yield $)$ of 3aa.

(1S,2S)-(2-Pyrrolidin-1-yl-1,2-dihydro-naphthalen-1-yl)-carbamic acid tert-butyl ester (3aa): $>99 \%$ ee by HPLC analysis with a chiral column (Chiralcel OD-H, hexane/2-propanol =90/10, $1.0 \mathrm{~mL} / \mathrm{min}), \mathrm{t}_{\mathrm{R}}(S)-(+), 9.4 \mathrm{~min}$ (major); $(R)-(-), 20.8 \mathrm{~min}$ (minor). $[\alpha]_{\mathrm{D}}^{25}+282.4\left(\mathrm{c} 1.10, \mathrm{CHCl}_{3}\right.$ ) for $\mathbf{3 a a}$ of $>99 \%$ ee. $\mathrm{mp} 84-87^{\circ} \mathrm{C}$; IR $(\mathrm{NaCl}$, $\left.\mathrm{cm}^{-1}\right)$ 3450, 2809, 2360, 1716, 1653, 1557, 1251, 1169, 1005. ${ }^{1} \mathrm{H}$ NMR $\left(\mathrm{CDCl}_{3}\right) \delta 1.42$ $(\mathrm{s}, 9 \mathrm{H}), 1.68(\mathrm{~m}, 4 \mathrm{H}), 2.59-2.69(\mathrm{~m}, 4 \mathrm{H}), 3.34(\mathrm{t}, J=6.0 \mathrm{~Hz}, 1 \mathrm{H}), 4.76(\mathrm{~d}, J=6.0 \mathrm{~Hz}$, 1H), 4.93 (bs, 1H), 5.97 (dd, $J=9.9,3.0 \mathrm{~Hz}, 1 \mathrm{H}), 6.60$ (d, $J=9.9 \mathrm{~Hz}, 1 \mathrm{H}), 7.06-7.08$ (m, $1 \mathrm{H}), 7.17-7.24(\mathrm{~m}, 2 \mathrm{H}), 7.31-7.33(\mathrm{~m}, 1 \mathrm{H}) ;{ }^{13} \mathrm{C} \mathrm{NMR}\left(\mathrm{CDCl}_{3}\right) \delta 155.2,134.9,132.2$, 
129.2, 128.4, 128.1, 126.8, 126.2, 61.2, 50.1, 28.4, 23.5. HRMS (EI) calcd for $\mathrm{C}_{19} \mathrm{H}_{26} \mathrm{~N}_{2} \mathrm{O}_{2}\left(\mathrm{M}^{+}\right)$: 314.1994. Found: 314.1991 .

(1S,2S)-(2-(Pyrrolidin-1-yl-1,2-dihydro-naphthalen-1-yl)-carbamic acid methyl ester (3ba): $63 \%$ ee by HPLC analysis with a chiral column (Chiralcel OD, hexane/2-propanol =90/10,1.0 mL/min), $\mathrm{t}_{\mathrm{R}}(R)-(-), 7.9 \mathrm{~min}$ (minor); $(S)-(+), 15.2 \mathrm{~min}$ (major). $[\alpha]_{\mathrm{D}}^{25}+229.0(\mathrm{c} 0.82, \mathrm{CHCl} 3)$ for $\mathbf{3 b a}$ of $63 \%$ ee. IR $\left(\mathrm{NaCl}, \mathrm{cm}^{-1}\right) 3317,2962$, $1718,1528,1451,1351,1256,1192,1119,1040,780 .{ }^{1} \mathrm{H} \mathrm{NMR}\left(\mathrm{CDCl}_{3}\right) \delta 1.67-1.71(\mathrm{~m}$, $4 \mathrm{H}), 2.58-2.68(\mathrm{~m}, 4 \mathrm{H}), 3.36(\mathrm{t}, J=4.8 \mathrm{~Hz}, 1 \mathrm{H}), 3.65(\mathrm{~s}, 3 \mathrm{H}), 4.91-4.96(\mathrm{~m}, 2 \mathrm{H}), 5.98$ $(\mathrm{dd}, J=9.9,4.8 \mathrm{~Hz}, 1 \mathrm{H}), 6.63(\mathrm{~d}, J=9.9 \mathrm{~Hz}, 1 \mathrm{H}), 7.08-7.11(\mathrm{~m}, 1 \mathrm{H}), 7.18-7.26(\mathrm{~m}, 2 \mathrm{H})$, $7.31(\mathrm{~d}, J=6.9 \mathrm{~Hz}, 1 \mathrm{H}) ;{ }^{13} \mathrm{C} \mathrm{NMR}\left(\mathrm{CDCl}_{3}\right) \delta 156.5,134.6,132.2,129.4,128.5,128.4$, 128.4, 126.9, 126.2, 61.2, 52.2, 50.9, 50.2, 23.6. HRMS calcd for $\mathrm{C}_{16} \mathrm{H}_{20} \mathrm{~N}_{2} \mathrm{O}_{2}\left(\mathrm{M}^{+}\right)$: 272.1524. Found: 272.1517.

(1S,2S)-(2-Pyrrolidin-1-yl-1,2-dihydro-naphthalen-1-yl)-carbamic acid benzyl ester (3ca): $68 \%$ ee by HPLC analysis with a chiral column (Chiralcel OD, hexane/2-propanol =90/10, $1.0 \mathrm{~mL} / \mathrm{min}), \mathrm{t}_{\mathrm{R}}(R)-(-), 7.9 \mathrm{~min}$ (minor); $(S)-(+), 9.7 \mathrm{~min}$ (major). $[\alpha]_{\mathrm{D}}^{25}+200.6\left(c 0.80, \mathrm{CHCl}_{3}\right)$ for $3 \mathrm{ca}$ of $68 \%$ ee; mp $90-95{ }^{\circ} \mathrm{C}$; IR $(\mathrm{NaCl}$, $\left.\mathrm{cm}_{-1}\right) 3318,2963,1711,1498,1453,1251,1118,1038,779,746,696 .{ }^{1} \mathrm{H}$ NMR $\left(\mathrm{CDCl}_{3}\right) \delta 1.70-1.88(\mathrm{~m}, 4 \mathrm{H}), 2.59-2.68(\mathrm{~m}, 4 \mathrm{H}), 3.37(\mathrm{t}, J=4.8 \mathrm{~Hz}, 1 \mathrm{H}), 4.99(\mathrm{bs}, 2 \mathrm{H})$, 5.05-5.12 (m, 2H), $5.97(\mathrm{dd}, J=9.9,4.8 \mathrm{~Hz}, 1 \mathrm{H}), 6.61(\mathrm{~d}, J=9.9 \mathrm{~Hz}, 1 \mathrm{H}), 7.07-7.32$ (m, $9 \mathrm{H}) ;{ }^{13} \mathrm{C} \mathrm{NMR}\left(\mathrm{CDCl}_{3}\right) \delta 155.8,136.6,134.6,132.2,129.3,128.6,128.5,128.4,128.3$, 128.2, 126.9, 126.2, 66.8, 61.2, 50.9, 50.2, 23.6. HRMS calcd for $\mathrm{C}_{22} \mathrm{H}_{24} \mathrm{~N}_{2} \mathrm{O}_{2}\left(\mathrm{M}^{+}\right)$: 348.1837. Found: 348.1836.

(1S,2S)- $N$-(2-pyrrolidin-1-yl-1,2-dihydro-naphthalen-1-yl)-benzamide (3da): $96 \%$ ee by HPLC analysis with a chiral column (Chiralcel OD-H, hexane/2-propanol $=90 / 10$, $1.0 \mathrm{~mL} / \mathrm{min}$ ), $\mathrm{t}_{\mathrm{R}}(R)-(-), 11.3 \mathrm{~min}$ (minor); $(S)-(+), 19.1 \mathrm{~min}$ (major). $[\alpha]_{\mathrm{D}}^{25}+372.4(c$ $\left.1.00, \mathrm{CHCl}_{3}\right)$ for $3 \mathrm{da}$ of $96 \%$ ee. $\mathrm{mp} 114-116{ }^{\circ} \mathrm{C}$; IR $\left(\mathrm{NaCl}, \mathrm{cm}^{-1}\right) 3299,3030,2963$, 2803, 1634, 1579, 1537, 1488, 1337, 1126, 779, 749, 706, 693. ${ }^{1} \mathrm{H}$ NMR $\left(\mathrm{CDCl}_{3}\right) \delta$ $1.69-1.73(\mathrm{~m}, 4 \mathrm{H}), 2.63-2.66(\mathrm{~m}, 2 \mathrm{H}), 2.76-2.79(\mathrm{~m}, 2 \mathrm{H}), 3.49(\mathrm{t}, J=4.0 \mathrm{~Hz}, 1 \mathrm{H}), 5.51$ (dd, $J=8.1,3.3 \mathrm{~Hz}, 1 \mathrm{H}), 6.05(\mathrm{dd}, J=9.6,4.5 \mathrm{~Hz}, 1 \mathrm{H}), 6.22(\mathrm{~d}, J=8.1 \mathrm{~Hz}, 1 \mathrm{H}), 6.69$ $(\mathrm{d}, J=9.9 \mathrm{~Hz}, 1 \mathrm{H}), 7.15(\mathrm{dd}, J=7.2,1.5 \mathrm{~Hz}, 1 \mathrm{H}), 7.19-7.30(\mathrm{~m}, 2 \mathrm{H}), 7.35-7.41(\mathrm{~m}, 3 \mathrm{H})$, $7.47(\mathrm{ddd}, J=7.2,6.0,1.5 \mathrm{~Hz}, 1 \mathrm{H}), 7.71(\mathrm{dd}, J=6.9,1.5 \mathrm{~Hz}, 2 \mathrm{H}) ;{ }^{13} \mathrm{C} \mathrm{NMR}\left(\mathrm{CDCl}_{3}\right) \delta$ $166.7,134.6,134.4,132.5,131.6,129.4,128.8,128.6,128.5,127.1,126.6,60.9,50.3$, 49.5, 23.7. HRMS calcd for $\mathrm{C}_{21} \mathrm{H}_{23} \mathrm{~N}_{2} \mathrm{O}\left[\mathrm{M}+\mathrm{H}^{+}\right]$: 319.1804 . Found: 319.1813 . 
(1S,2S)- $N$-(2-pyrrolidin-1-yl-1,2-dihydro-naphthalen-1-yl)-acetamide (3ea): $99 \%$ ee by HPLC analysis with a chiral column (Chiralcel OD, hexane/2-propanol = 90/10, $1.0 \mathrm{~mL} / \mathrm{min}), \mathrm{t}_{\mathrm{R}}(R)-(-), 12.5 \mathrm{~min}$ (minor); $(S)-(+), 14.6 \mathrm{~min}$ (major). $[\alpha]^{25}{ }_{\mathrm{D}}+313.0(c$ $1.00, \mathrm{CHCl}_{3}$ ) for 3ea of $99 \%$ ee. IR $\left(\mathrm{NaCl}, \mathrm{cm}^{-1}\right)$ 3279, 2966, 2236, 1650, 1537, 1451, 1371, 1293, 1128, 999, 923, 910, 781, 731. ${ }^{1} \mathrm{H}$ NMR $\left(\mathrm{CDCl}_{3}\right) \delta 1.66-1.69$ (m, 4H), 1.91 (s, 3H), 2.54-2.59 (m, 2H), 2.66-2.72 (m, 2H), 3.33 (t, $J=4.2 \mathrm{~Hz}, 1 \mathrm{H}), 5.29$ (dd, $J=8.2$, $3.2 \mathrm{~Hz}, 1 \mathrm{H}), 5.79$ (d, $J=8.0 \mathrm{~Hz}, 1 \mathrm{H}), 5.98$ (dd, $J=9.9,4.8 \mathrm{~Hz}, 1 \mathrm{H}), 6.63$ (d, $J=9.6 \mathrm{~Hz}$, $1 \mathrm{H}), 7.09-7.11(\mathrm{~m}, 1 \mathrm{H}), 7.19-7.28(3 \mathrm{H}) ;{ }^{13} \mathrm{C} \mathrm{NMR}\left(\mathrm{CDCl}_{3}\right) \delta 169.1,134.5,132.2,129.2$, 128.6, 128.3, 128.2, 126.8, 126.3, 60.6, 50.0, 48.9, 23.5, 23.4. HRMS calcd for $\mathrm{C}_{16} \mathrm{H}_{20} \mathrm{~N}_{2} \mathrm{O}\left(\mathrm{M}^{+}\right): 254.1419$. Found: 254.1411 .

\section{(1S,2S)- $N$-Phenyl-(2-pyrrolidin-1-yl-1,2-dihydro-naphthalen-1-yl)amine (3fa):}

$25 \%$ ee by HPLC analysis with a chiral column (Chiralcel OD, hexane/2-propanol = 95/5, $1.0 \mathrm{~mL} / \mathrm{min}), \mathrm{t}_{\mathrm{R}}(R)-(-), 4.9 \mathrm{~min}$ (minor); $(S)-(+), 5.4 \mathrm{~min}$ (major). $[\alpha]^{25}{ }_{\mathrm{D}}+58.6(c$ $\left.1.22, \mathrm{CHCl}_{3}\right)$ for $\mathbf{3 f a}$ of $25 \%$ ee. IR $\left(\mathrm{NaCl}, \mathrm{cm}^{-1}\right) 3332,2963,2361,2343,1722,1600$, $1501,1451,1430,1314,1261,1123,1056,1032,992,908,749,693 .{ }^{1} \mathrm{H}$ NMR $\left(\mathrm{CDCl}_{3}\right)$ $\delta 1.72(\mathrm{~m}, 4 \mathrm{H}), 2.60-2.65(\mathrm{~m}, 4 \mathrm{H}), 3.73(\mathrm{bs}, 1 \mathrm{H}), 4.12(\mathrm{bs}, 1 \mathrm{H}), 4.58(\mathrm{bs}, 1 \mathrm{H}), 6.03$ (dd, $J=9.9,4.4 \mathrm{~Hz}, 1 \mathrm{H}), 6.68-6.74(\mathrm{~m}, 4 \mathrm{H}), 7.11-7.29(\mathrm{~m}, 6 \mathrm{H}) ;{ }^{13} \mathrm{C} \mathrm{NMR}\left(\mathrm{CDCl}_{3}\right) \delta 147.9$, $136.4,132.5,129.4,128.1,127.9,126.7,117.8,113.8,58.7,55.5,49.0$, 23.7. HRMS calcd for $\mathrm{C}_{20} \mathrm{H}_{22} \mathrm{~N}_{2}\left(\mathrm{M}^{+}\right)$: 290.1782. Found: 290.1783 .

\section{(1S,2S)-(2-pyrrolidin-1-yl-1,2-dihydronaphthalen-1-yl)-p-toluenesulfonylamine}

(3ha): $10 \%$ ee by HPLC analysis with a chiral column (Chiralcel OD, hexane/2-propanol = 90/10, $1.0 \mathrm{~mL} / \mathrm{min}), \mathrm{t}_{\mathrm{R}}(R)-(-), 7.9 \mathrm{~min}$ (minor); $(S)-(+), 9.7 \mathrm{~min}$ (major). $[\alpha]^{25}+31.3\left(c 1.22, \mathrm{CHCl}_{3}\right.$ ) for 3 ha of $10 \%$ ee; mp $140-145{ }^{\circ} \mathrm{C}$; IR $(\mathrm{NaCl}$, $\left.\mathrm{cm}^{-1}\right)$ 3496, 3035, 2967, 1454, 1193, 1117, 1048. ${ }^{1} \mathrm{H}$ NMR $\left(\mathrm{CDCl}_{3}\right) \delta 1.58-1.63(\mathrm{~m}, 4 \mathrm{H})$, 2.29-2.36 (m, 2H), 2.45 (s, 3H), 2.49-2.58 (m, 2H), 3.89 (d, J=4.2 Hz, 1H), 4.45 (d, $J=$ $3.7 \mathrm{~Hz}, 1 \mathrm{H}), 4.70(\mathrm{bs}, 1 \mathrm{H}), 5.93(\mathrm{dd}, J=9.9,4.8 \mathrm{~Hz}, 1 \mathrm{H}), 6.61$ (d, $J=9.9 \mathrm{~Hz}, 1 \mathrm{H}), 6.84$ $(\mathrm{d}, J=7.6 \mathrm{~Hz}, 1 \mathrm{H}), 7.02-7.08(\mathrm{~m}, 2 \mathrm{H}), 7.17-7.22(\mathrm{~m}, 1 \mathrm{H}), 7.30(\mathrm{~d}, J=8.4 \mathrm{~Hz}, 2 \mathrm{H}), 7.74$ $(\mathrm{d}, J=8.4 \mathrm{~Hz}, 2 \mathrm{H}) ;{ }^{13} \mathrm{C} \mathrm{NMR}\left(\mathrm{CDCl}_{3}\right) \delta 143.4,138.0,133.6,132.1,129.7,129.4,128.5$, 128.2, 128.1, 127.4, 126.9, 125.5, 61.2, 53.6, 49.9, 23.4, 21.6. Anal. Calcd for $\mathrm{C}_{21} \mathrm{H}_{24} \mathrm{~N}_{2} \mathrm{O}_{2} \mathrm{~S}: \mathrm{C}, 68.45 ; \mathrm{H}, 6.56$; N, 7.60. Found: C, 68.51; H, 6.62; N, 7.55.

Rhodium-Catalyzed Asymmetric Ring-Opening of 1a with Piperidine (2b) under the neat reaction conditions. The reaction conditions and results are summarized in Tables, 3-6. A typical procedure is given for the reaction of $1 \mathbf{a}$ with 5.0 eq. of piperidine 
(2b) in the presence of $2.5 \mathrm{~mol} \%$ of $[\mathrm{Rh}(\mathrm{cod}) \mathrm{Cl}]_{2}$ and $11.0 \mathrm{~mol} \%$ of $4 \mathbf{a}$ (entry 1 in Table $3)$.

To a $10 \mathrm{~mL}$ round bottom flask were added $1 \mathrm{a}(80 \mathrm{mg}, 0.33 \mathrm{mmol}),[\mathrm{Rh}(\mathrm{cod}) \mathrm{Cl}]_{2}(4$ $\mathrm{mg}, 8 \mu \mathrm{mol})$, and $4 \mathbf{a}(26 \mathrm{mg}, 36 \mu \mathrm{mol})$ under nitrogen atmosphere. Then, piperidine $(0.17 \mathrm{~mL}, 1.66 \mathrm{mmol})$ was added and the mixture was allowed to stir at $80{ }^{\circ} \mathrm{C}$ for $60 \mathrm{~h}$. The resulting mixture was cooled to room temperature and diluted in $\mathrm{CH}_{2} \mathrm{Cl}_{2}$. The black solution was filtered through Celite and the filtrate was concentrated under reduced pressure. The residue was purified by flash column chromatography (hexane/ethyl acetate $=70 / 30)$ to give $90 \mathrm{mg}(83 \%$ yield $)$ of $\mathbf{3 a b}$ as a semisolid.

(1S,2S)-(2-Piperidin-1-yl-1,2-dihydro-naphthalen-1-yl)-carbamic acid tert-butyl ester (3ab): $97 \%$ ee by HPLC analysis with a chiral column (Chiralcel OD-H, hexane/2-propanol =90/10, $1.0 \mathrm{~mL} / \mathrm{min}), \mathrm{t}_{\mathrm{R}}(S)-(+), 9.8 \mathrm{~min}$ (major); $(R)-(-), 25.1 \mathrm{~min}$ (minor). $[\alpha]_{\mathrm{D}}^{25}+261.0\left(c 1.10, \mathrm{CHCl}_{3}\right)$ for $\mathbf{3 a b}$ of $97 \%$ ee. mp $118-122{ }^{\circ} \mathrm{C}$; IR $(\mathrm{NaCl}$, $\left.\mathrm{cm}^{-1}\right) 3448,2978,2288,1713,1549,1251,1170,1005,760 .{ }^{1} \mathrm{H} \mathrm{NMR}\left(\mathrm{CDCl}_{3}\right) \delta 1.45(\mathrm{~s}$, $9 \mathrm{H}), 1.37-1.50(\mathrm{~m}, 6 \mathrm{H}), 2.42-2.44(\mathrm{~m}, 2 \mathrm{H}), 2.56-2.61(\mathrm{~m}, 2 \mathrm{H}), 3.37(\mathrm{t}, \mathrm{J}=6.0 \mathrm{~Hz}, 1 \mathrm{H})$, 4.60 (bs, 1H), 5.03 (bs, 1H), 5.97 (dd, $J=9.9,6.0 \mathrm{~Hz}, 1 \mathrm{H}), 6.62$ (d, $J=9.9 \mathrm{~Hz}, 1 \mathrm{H})$, 7.06-7.09 (m, 1H), 7.19-7.23 (m, 2H), 7.34-7.36 (m, 1H); ${ }^{13} \mathrm{C} \mathrm{NMR}\left(\mathrm{CDCl}_{3}\right) \delta$ 155.3, 135.8, 132.6, 129.4, 128.2, 128.0, 127.8, 126.8, 126.4, 64.9, 50.1, 48.1, 29.8, 28.6, 26.6, 24.7. HRMS (EI) calcd for $\mathrm{C}_{20} \mathrm{H}_{28} \mathrm{~N}_{2} \mathrm{O}_{2}\left(\mathrm{M}^{+}\right)$: 328.2150 . Found: 328.2145 .

(1S,2S)-[2-(4-Phenyl-piperazin-1-yl)-1,2-dihydro-naphthalen-1-yl]-carbamic acid tert-butyl ester (3ac): $92 \%$ ee by HPLC analysis with a chiral column (Chiralcel AD, hexane/2-propanol =90/10, $0.5 \mathrm{~mL} / \mathrm{min}), \mathrm{t}_{\mathrm{R}}(R)-(-), 7.2 \mathrm{~min}$ (minor); $(S)-(+), 8.5 \mathrm{~min}$ (major). $[\alpha]_{\mathrm{D}}^{25}+319.1$ (c 1.00, $\mathrm{CHCl}_{3}$ ) for 3ac of $94 \%$ ee. mp $166-167{ }^{\circ} \mathrm{C}$; IR $(\mathrm{NaCl}$, $\left.\mathrm{cm}^{-1}\right) 3453,2979,2328,1711,1562,1483,1367,1169 .{ }^{1} \mathrm{H} \mathrm{NMR}\left(\mathrm{CDCl}_{3}\right) \delta 1.44(\mathrm{~s}, 9 \mathrm{H})$, 2.68-2.70 (m, 2H), 2.76-2.82 (m, 2H), $3.10(\mathrm{t}, J=4.8 \mathrm{~Hz}, 4 \mathrm{H}), 3.47(\mathrm{t}, J=6.0 \mathrm{~Hz}, 1 \mathrm{H})$, $4.60(\mathrm{~d}, J=6.0 \mathrm{~Hz}, 1 \mathrm{H}), 5.06(\mathrm{bs}, 1 \mathrm{H}), 6.00(\mathrm{dd}, J=9.9,6.0 \mathrm{~Hz}, 1 \mathrm{H}), 6.66(\mathrm{~d}, J=9.9$ $\mathrm{Hz}, 1 \mathrm{H}), 6.82(\mathrm{t}, J=7.2 \mathrm{~Hz}, 1 \mathrm{H}), 6.86(\mathrm{~d}, J=8.4 \mathrm{~Hz}, 2 \mathrm{H}), 7.09-7.11(\mathrm{~m}, 1 \mathrm{H}), 7.25(\mathrm{~m}$, $4 \mathrm{H}), 7.37(\mathrm{~m}, 1 \mathrm{H}) ;{ }^{13} \mathrm{C} \mathrm{NMR}\left(\mathrm{CDCl}_{3}\right) \delta 155.4,151.6,135.4,132.5,129.8,129.2,128.4$, $128.3,127.9,126.9,125.5,119.7,116.2$, 79.6, 64.0, 49.8, 48.8, 48.6, 29.8, 28.6. HRMS (EI) calcd for $\mathrm{C}_{25} \mathrm{H}_{31} \mathrm{~N}_{3} \mathrm{O}_{2}\left(\mathrm{M}^{+}\right)$: 405.2416. Found: 405.2411.

(1S,2S)-(2-Morpholin-4-yl-1,2-dihydro-naphthalen-1-yl)-carbamic acid tert-butyl ester (3ad): $97 \%$ ee by HPLC analysis with a chiral column (Chiralcel AD, hexane/2-propanol =90/10, $0.5 \mathrm{~mL} / \mathrm{min}$ ), $\mathrm{t}_{\mathrm{R}}(S)-(+), 15.3 \mathrm{~min}$ (major); $(R)-(-), 16.7 \mathrm{~min}$ (minor). $[\alpha]_{\mathrm{D}}^{25}+337.7\left(c 1.00, \mathrm{CHCl}_{3}\right)$ for $\mathbf{3 a d}$ of $97 \%$ ee. $\mathrm{mp} 167-168{ }^{\circ} \mathrm{C}$; IR $(\mathrm{NaCl}$, 
$\left.\mathrm{cm}^{-1}\right) 3448,2978,2281,1714,1550,1367,1250,1170,1005,757 .{ }^{1} \mathrm{H}$ NMR $\left(\mathrm{CDCl}_{3}\right) \delta$ 1.45 (s, 9H), 2.52-2.54 (m, 2H), 2.62-2.65 (m, 2H), $3.36(\mathrm{t}, J=4.8 \mathrm{~Hz}, 1 \mathrm{H}), 3.61-3.62$ (m, 4H), 4.59 (d, $J=6.0 \mathrm{~Hz}, 1 \mathrm{H}), 5.02$ (bs, 1H), 5.97 (dd, $J=9.9,6.0 \mathrm{~Hz}, 1 \mathrm{H}), 6.65$ (dd, $J=9.9,1.2 \mathrm{~Hz}, 1 \mathrm{H}), 7.08-7.12(\mathrm{~m}, 1 \mathrm{H}), 7.21-7.28(\mathrm{~m}, 2 \mathrm{H}), 7.35-7.37(\mathrm{~m}, 1 \mathrm{H}) ;{ }^{13} \mathrm{C}$ NMR $\left(\mathrm{CDCl}_{3}\right) \delta 155.2,135.1,132.3,129.7,128.2,128.1,127.7,126.7,125.1,79.5$, 67.3, 64.2, 49.2, 48.6, 28.4. Anal. Cacld for $\mathrm{C}_{19} \mathrm{H}_{26} \mathrm{~N}_{2} \mathrm{O}_{3}$ : C, 69.06; H, 7.93; N, 8.48. Found: C, 68.86; H, 7.97; N, 8.39.

(1S,2S)-(2-Dibenzylamino-1,2-dihydro-naphthalen-1-yl)-carbamic acid tert-butyl ester (3ae): $97 \%$ ee by HPLC analysis with a chiral column (Chiralcel OD, hexane/2-propanol =99/1, $0.5 \mathrm{~mL} / \mathrm{min}), \mathrm{t}_{\mathrm{R}}(S)-(+), 15.4 \mathrm{~min}$ (major); $(R)-(-), 17.0 \mathrm{~min}$ (minor). $[\alpha]^{25}{ }_{\mathrm{D}}+150.4\left(c 1.00, \mathrm{CHCl}_{3}\right)$ for $\mathbf{3 a e}$ of $97 \%$ ee. ${ }^{1} \mathrm{H} \mathrm{NMR}\left(\mathrm{CDCl}_{3}\right) \delta 1.53(\mathrm{~s}$, 9H), 3.43-3.50 (m, 1H), 3.55 (d, $J=13.5 \mathrm{~Hz}, 2 \mathrm{H}), 3.74$ (d, $J=13.5 \mathrm{~Hz}, 2 \mathrm{H}), 4.48$ (d, $J$ $=9.0 \mathrm{~Hz}, 1 \mathrm{H}), 5.24(\mathrm{t}, J=9.0 \mathrm{~Hz}, 1 \mathrm{H}), 6.05(\mathrm{dd}, J=9.9,6.0 \mathrm{~Hz}, 1 \mathrm{H}), 6.59(\mathrm{dd}, J=9.9$, $1.8 \mathrm{~Hz}, 1 \mathrm{H}), 7.02-7.05(\mathrm{~m}, 1 \mathrm{H}), 7.18-7.33(\mathrm{~m}, 9 \mathrm{H}), 7.37$ (d, $J=6.9 \mathrm{~Hz}, 4 \mathrm{H}) ;{ }^{13} \mathrm{C} \mathrm{NMR}$ $\left(\mathrm{CDCl}_{3}\right) \delta 155.7,140.0,135.8,132.6,129.5,129.2,129.1,128.3,128.1,127.9,127.4$, 127.0, 126.9, 126.7, 79.5, 59.5, 54.0, 49.4, 28.6, 28.5. HRMS (EI) calcd for $\mathrm{C}_{29} \mathrm{H}_{32} \mathrm{~N}_{2} \mathrm{O}_{2}$ $\left(\mathrm{M}^{+}\right)$: 440.2463. Found: 440.2455.

\section{(1S,2S)-[2-(N-Methyl- $N$-phenyl-amino)-1,2-dihydro-naphthalen-1-yl]-carbamic}

acid tert-butyl ester (3af): $97 \%$ ee by HPLC analysis with a chiral column (Chiralcel $\mathrm{AD}$, hexane/2-propanol =98/2, $1.0 \mathrm{~mL} / \mathrm{min}$ ), $\mathrm{t}_{\mathrm{R}}(R)-(-), 11.5 \mathrm{~min}$ (minor); $(S)-(+), 13.4$ min (major). $[\alpha]^{25}+35.6$ (c 1.00, $\mathrm{CHCl}_{3}$ ) for 3af of $97 \%$ ee. mp 94-96 ${ }^{\circ} \mathrm{C}$; IR $(\mathrm{NaCl}$, $\left.\mathrm{cm}^{-1}\right) 34042973,1717,1595,1502,1369,1108,1032,991,864,811,753 .{ }^{1} \mathrm{H}$ NMR $\left(\mathrm{CDCl}_{3}\right) \delta 1.33(\mathrm{~s}, 9 \mathrm{H}), 2.82(\mathrm{~s}, 3 \mathrm{H}), 4.51(\mathrm{~d}, J=9.0 \mathrm{~Hz}, 1 \mathrm{H}), 4.77(\mathrm{~d}, J=9.0 \mathrm{~Hz}, 1 \mathrm{H})$, $5.19(\mathrm{t}, J=9.0 \mathrm{~Hz}, 1 \mathrm{H}), 5.90(\mathrm{dd}, J=9.9,3.0 \mathrm{~Hz}, 1 \mathrm{H}), 6.58(\mathrm{dd}, J=9.9,3.0 \mathrm{~Hz}, 1 \mathrm{H})$, $6.71(\mathrm{t}, J=6.9 \mathrm{~Hz}, 1 \mathrm{H}), 6.83(\mathrm{~d}, J=8.2 \mathrm{~Hz}, 2 \mathrm{H}), 7.08(\mathrm{~d}, J=7.6 \mathrm{~Hz}, 1 \mathrm{H}), 7.18-7.22(\mathrm{~m}$, $1 \mathrm{H}) ;{ }^{13} \mathrm{C} \mathrm{NMR}\left(\mathrm{CDCl}_{3}\right) \delta 155.5,150.2,135.9,132.8,130.1,129.9,129.2,128.0,127.9$, 126.7, 125.8, 117.1, 113.7, 79.4, 60.8, 52.4, 33.1, 28.3. HRMS (EI) calcd for $\mathrm{C}_{22} \mathrm{H}_{26} \mathrm{~N}_{2} \mathrm{O}_{2}\left(\mathrm{M}^{+}\right)$: 350.1994. Found: 350.1999 .

\section{(1S,2S)-[2-(3,4-Dihydro-2H-quinolin-1-yl)-1,2-dihydronaphthalen-1-yl]-carbamic}

acid tert-butyl ester (3ag): $97 \%$ ee by HPLC analysis with a chiral column (Chiralcel $\mathrm{AD}$, hexane/2-propanol =95/5, $1.0 \mathrm{~mL} / \mathrm{min}), \mathrm{t}_{\mathrm{R}}(S)-(+), 7.5 \mathrm{~min}$ (major); $(R)-(-), 8.4$ min (minor). $[\alpha]^{25}+269.3\left(c \quad 0.50, \mathrm{CHCl}_{3}\right.$ ) for $\mathbf{3 a g}$ of $97 \%$ ee. $\mathrm{mp} 155-160{ }^{\circ} \mathrm{C}$; IR $\left(\mathrm{NaCl}, \mathrm{cm}^{-1}\right) 3315,2929,1691,1601,1502,1453,1365,1169,1048,877,784 .{ }^{1} \mathrm{H}$ $\operatorname{NMR}\left(\mathrm{CDCl}_{3}\right) \delta 1.35(\mathrm{~s}, 9 \mathrm{H}), 1.81-1.91(\mathrm{~m}, 2 \mathrm{H}), 2.72(\mathrm{t}, J=6.4 \mathrm{~Hz}, 2 \mathrm{H}), 3.21(\mathrm{t}, J=$ 
$5.2 \mathrm{~Hz}, 2 \mathrm{H}), 4.59$ (d, $J=9.0 \mathrm{~Hz}, 1 \mathrm{H}), 4.79(\mathrm{~d}, J=9.0 \mathrm{~Hz}, 1 \mathrm{H}), 5.25$ (t, $J=9.0 \mathrm{~Hz}, 1 \mathrm{H})$, 5.89-5.92 (m, 1H), 6.56-6.63 (m, 2H), $6.77(\mathrm{~d}, J=8.4 \mathrm{~Hz}, 1 \mathrm{H}), 6.95(\mathrm{~d}, J=7.2 \mathrm{~Hz}, 1 \mathrm{H})$, 7.00-7.04 (m, 1H), 7.09-7.11 (m, 1H), 7.20-7.24 (m, 2H), $7.35(\mathrm{~d}, J=7.6 \mathrm{~Hz}, 1 \mathrm{H}) ;{ }^{13} \mathrm{C}$ NMR $\left(\mathrm{CDCl}_{3}\right) \delta 155.6,145.5,136.1,132.9,130.3,129.6,129.6,128.1,127.9,127.1$, $126.8,126.1,123.8,116.2,111.3,79.6,58.5,51.8,43.7,28.5,28.4,22.5$. HRMS (EI) calcd for $\mathrm{C}_{24} \mathrm{H}_{28} \mathrm{~N}_{2} \mathrm{O}_{2}\left(\mathrm{M}^{+}\right)$: 376.2150. Found: 376.2159 .

\section{(1S,2S)-[2-Phenylamino-1,2-dihydronaphthalen-1-yl]-carbamic acid tert-butyl} ester (3ah): $96 \%$ ee by HPLC analysis with a chiral column (Chiralcel OD, hexane/2-propanol = 98/2, $1.0 \mathrm{~mL} / \mathrm{min}), \mathrm{t}_{\mathrm{R}}(R)-(-), 16.2 \mathrm{~min}$ (minor); $(S)-(+), 19.8 \mathrm{~min}$ (major). $[\alpha]^{25}+175.0\left(c\right.$ 1.00, $\mathrm{CHCl}_{3}$ ) for $\mathbf{3 a h}$ of $96 \%$ ee. mp 91-96 ${ }^{\circ} \mathrm{C}$; IR $(\mathrm{NaCl}$, $\mathrm{cm}^{-1}$ ) 3396, 2976, 1701, 1601, 1503, 1366, 1323, 1248, 1167, 1046, 781, 748, 692. ${ }^{1} \mathrm{H}$ NMR $\left(\mathrm{CDCl}_{3}\right) \delta 1.45(\mathrm{~s}, 9 \mathrm{H}), 3.82-3.97(\mathrm{~m}, 1 \mathrm{H}), 4.22-4.30(\mathrm{~m}, 1 \mathrm{H}), 4.69(\mathrm{~d}, J=9.0 \mathrm{~Hz}$, $1 \mathrm{H}), 5.04$ (t, $J=9.0 \mathrm{~Hz}, 1 \mathrm{H}), 6.09$ (dd, $J=9.9,6.0 \mathrm{~Hz}, 1 \mathrm{H}), 6.57$ (d, $J=9.9 \mathrm{~Hz}, 1 \mathrm{H})$, $6.71(\mathrm{~d}, J=8.1 \mathrm{~Hz}, 3 \mathrm{H}), 7.12-7.33(\mathrm{~m}, 6 \mathrm{H}) ;{ }^{13} \mathrm{C} \mathrm{NMR}\left(\mathrm{CDCl}_{3}\right) \delta 156.1,146.9,134.2$, 132.6, 129.6, 128.7, 128.6, 128.4, 128.1, 127.1, 117.7, 113.3, 80.0, 53.6, 52.5, 28.6. HRMS (EI) calcd for $\mathrm{C}_{24} \mathrm{H}_{30} \mathrm{~N}_{2} \mathrm{O}_{4}\left(\mathrm{M}^{+}\right)$: 336.1837 . Found: 336.1837 .

(1S,2S)-[2-(Naphthalen-1-ylamino)-1,2-dihydro-naphthalen-1-yl]-carbamic acid tert-butyl ester (3ai): 97\% ee by HPLC analysis with a chiral column (Chiralcel OD, hexane/2-propanol =98/2,1.0 mL/min), $\mathrm{t}_{\mathrm{R}}(R)-(-), 20.5 \mathrm{~min}$ (minor); $(S)-(+), 37.1 \mathrm{~min}$ (major). $[\alpha]_{\mathrm{D}}^{25}+213.5\left(c 1.00, \mathrm{CHCl}_{3}\right)$ for 3ai of $97 \%$ ee. IR $\left(\mathrm{NaCl}, \mathrm{cm}^{-1}\right) 3419,2975$, 1696, 1582, 1504, 1485, 1410, 1247, 1165, 1047, 909, 784, 733. ${ }^{1} \mathrm{H}$ NMR $\left(\mathrm{CDCl}_{3}\right) \delta$ $1.43(\mathrm{~s}, 9 \mathrm{H}), 4.40(\mathrm{~d}, J=9.0 \mathrm{~Hz}, 1 \mathrm{H}), 4.84(\mathrm{~d}, J=9.0 \mathrm{~Hz}, 1 \mathrm{H}), 4.97$ (s, 1H), 5.29 (t, $J=$ $9.9 \mathrm{~Hz}, 1 \mathrm{H}), 6.15(\mathrm{dd}, J=9.9,6.0 \mathrm{~Hz}, 1 \mathrm{H}), 6.54$ (d, $J=9.9 \mathrm{~Hz}, 1 \mathrm{H}), 6.73$ (d, $J=7.6 \mathrm{~Hz}$, $1 \mathrm{H}), 7.12$ (d, $J=7.2 \mathrm{~Hz}, 1 \mathrm{H}), 7.19-7.27$ (m, 3H), 7.32-7.41 (m, 4H), 7.72-7.77 (m, 2H);

${ }^{13} \mathrm{C} \mathrm{NMR}\left(\mathrm{CDCl}_{3}\right) \delta 156.8,142.2,134.6,134.5,133.1,129.1,128.6,128.5,128.2$, 127.0, 126.8, 126.3, 125.8, 124.7, 123.5, 120.5, 117.3, 104.2, 80.3, 55.3, 52.9, 28.5 . HRMS (EI) calcd for $\mathrm{C}_{25} \mathrm{H}_{26} \mathrm{~N}_{2} \mathrm{O}_{2}\left(\mathrm{M}_{+}\right)$: 386.1994. Found: 386.2002 .

(1S,2S)-[2-(1H-Indol-3-yl)-1,2-dihydro-naphthalen-1-yl]-carbamic acid tert-butyl ester (3aj): $96 \%$ ee by HPLC analysis with a chiral column (Chiralcel AD, hexane/2-propanol =90/10, $1.0 \mathrm{~mL} / \mathrm{min}$ ), $\mathrm{t}_{\mathrm{R}}(S)-(+), 13.0 \mathrm{~min}$ (major); $(R)-(-), 15.5 \mathrm{~min}$ (minor). $[\alpha]^{25}+133.8\left(c 0.80, \mathrm{CHCl}_{3}\right)$ for 3aj of $96 \%$ ee. IR $\left(\mathrm{NaCl}, \mathrm{cm}^{-1}\right) 3412,3339$, 2977, 1690, 1489, 1366, 1166, 1043, 1020, 908, 740. ${ }^{1} \mathrm{H}$ NMR $\left(\mathrm{CDCl}_{3}\right) \delta 1.41(\mathrm{~s}, 9 \mathrm{H})$, $4.19(\mathrm{~s}, 1 \mathrm{H}), 4.91(\mathrm{~d}, J=8.4 \mathrm{~Hz}, 1 \mathrm{H}), 5.08(\mathrm{dd}, J=8.8,4.8 \mathrm{~Hz}, 1 \mathrm{H}), 6.15(\mathrm{dd}, J=9.9$, $6.0 \mathrm{~Hz}, 1 \mathrm{H}), 6.64(\mathrm{~d}, J=9.9 \mathrm{~Hz}, 1 \mathrm{H}), 6.72(\mathrm{~s}, 1 \mathrm{H}), 7.11-7.23(\mathrm{~m}, 8 \mathrm{H}), 7.92(\mathrm{~m}, 2 \mathrm{H}) ;{ }^{13} \mathrm{C}$ 
NMR $\left(\mathrm{CDCl}_{3}\right) \delta 155.2,136.7,134.1,132.8,129.9,128.8,128.3,127.9,127.1,126.9$, $126.5,122.1,119.7,119.5,113.9,111.2,79.4,53.5,38.5,28.5$. HRMS calcd for $\mathrm{C}_{23} \mathrm{H}_{24} \mathrm{~N}_{2} \mathrm{O}_{2}\left(\mathrm{M}^{+}\right)$: 302.1055. Found: 302.1062 .

\section{(1S,2S)-(2-Piperidin-1-yl-1,2-dihydro-5,8-dimethyl-naphthalen-1-yl)-carbamic}

acid tert-butyl ester (3ib): 94\% ee by HPLC analysis with a chiral column (Chiralcel OD-H, hexane/2-propanol = 98/2, $1.0 \mathrm{~mL} / \mathrm{min}), \mathrm{t}_{\mathrm{R}}(R)-(-), 11.5 \mathrm{~min}$ (minor); $(S)-(+)$, 12.9 min (major). $[\alpha]_{\mathrm{D}}^{25}+206.4\left(c 1.00, \mathrm{CHCl}_{3}\right)$ for 3 ib of $94 \%$ ee. $\mathrm{mp} 143-145{ }^{\circ} \mathrm{C}$; IR $\left(\mathrm{NaCl}, \mathrm{cm}^{-1}\right)$ 3178, 2927, 2853, 1695, 1521, 1459, 1363, 1310, 1248, 1166, 1097, 1016, 992, 921, 859, 804, 732. ${ }^{1} \mathrm{H}$ NMR $\left(\mathrm{CDCl}_{3}\right) \delta 1.33-1.57(\mathrm{~m}, 15 \mathrm{H}), 2.14-2.38(\mathrm{~m}, 8 \mathrm{H})$, 2.52-2.70 (m, 2H), $3.35(\mathrm{~d}, J=6.0 \mathrm{~Hz}, 1 \mathrm{H}), 4.39$ (d, $J=6.0 \mathrm{~Hz}, 1 \mathrm{H}), 5.15$ (d, $J=8.1 \mathrm{~Hz}$, $1 \mathrm{H}), 5.95(\mathrm{dd}, J=9.9,6.0 \mathrm{~Hz}, 1 \mathrm{H}), 6.90(\mathrm{~d}, J=9.9 \mathrm{~Hz}, 1 \mathrm{H}), 6.98(\mathrm{~s}, 2 \mathrm{H}) ;{ }^{13} \mathrm{C} \mathrm{NMR}$ $\left(\mathrm{CDCl}_{3}\right) \delta 155.1,133.8,133.1,131.8,130.5,130.1,129.7,126.6,125.1,79.5,64.0,50.2$, 43.5, 28.6, 28.4, 26.6, 24.8, 19.1, 18.7. HRMS (EI) calcd for $\mathrm{C}_{22} \mathrm{H}_{32} \mathrm{~N}_{2} \mathrm{O}_{2}\left(\mathrm{M}^{+}\right)$: 356.2460. Found: 356.2463 .

(1S,2S)-[2-(4-Phenyl-piperazin-1-yl)-1,2-dihydro-5,8-dimethyl-naphthalen-1-yl]-c arbamic acid tert-butyl ester (3ic): $94 \%$ ee by HPLC analysis with a chiral column (Chiralcel AD, hexane/2-propanol =90/10, $0.5 \mathrm{~mL} / \mathrm{min}$ ), $\mathrm{t}_{\mathrm{R}}(R)-(-), 10.1 \mathrm{~min}$ (minor); $(S)-(+), 11.6 \mathrm{~min}$ (major). $[\alpha]_{\mathrm{D}}^{25}+325.4\left(c 1.00, \mathrm{CHCl}_{3}\right)$ for 3ic of $94 \%$ ee. mp 147-149 ${ }^{\circ} \mathrm{C}$; IR $\left(\mathrm{NaCl}, \mathrm{cm}^{-1}\right)$ 3194, 2971, 1703, 1598, 1501, 1449, 1363, 1237, 1162, 998, 755. ${ }^{1} \mathrm{H} \mathrm{NMR}\left(\mathrm{CDCl}_{3}\right) \delta 1.43(\mathrm{~s}, 9 \mathrm{H}), 2.33(\mathrm{~s}, 3 \mathrm{H}), 2.35(\mathrm{~s}, 3 \mathrm{H}), 2.48-2.52(\mathrm{~m}, 2 \mathrm{H})$, 2.82-2.87 (m, 2H), $3.07(\mathrm{t}, J=4.8 \mathrm{~Hz}, 4 \mathrm{H}), 3.46(\mathrm{~d}, J=6.0 \mathrm{~Hz}, 1 \mathrm{H}), 4.41(\mathrm{~d}, J=8.1 \mathrm{~Hz}$, $1 \mathrm{H}), 5.17(\mathrm{~d}, J=8.1 \mathrm{~Hz}, 1 \mathrm{H}), 5.97(\mathrm{dd}, J=9.9,6.0 \mathrm{~Hz}, 1 \mathrm{H}), 6.78-6.86(\mathrm{~m}, 3 \mathrm{H}), 6.96(\mathrm{~d}$, $J=9.9 \mathrm{~Hz}, 1 \mathrm{H}), 7.00(\mathrm{~s}, 2 \mathrm{H}), 7.21(\mathrm{t}, J=8.6 \mathrm{~Hz}, 2 \mathrm{H}) ;{ }^{13} \mathrm{C} \mathrm{NMR}\left(\mathrm{CDCl}_{3}\right) \delta 155.2,151.6$, 134.1, 132.7, 132.0, 130.4, 130.0, 129.2, 127.1, 124.5, 119.7, 116.2, 79.6, 62.8, 49.7, 48.9, 44.1, 28.6, 19.1, 18.7. HRMS (EI) calcd for $\mathrm{C}_{27} \mathrm{H}_{35} \mathrm{~N}_{3} \mathrm{O}_{2}\left(\mathrm{M}^{+}\right)$: 443.2736. Found: 443.2729 .

\section{(1S,2S)-(2-Morpholin-4-yl-1,2-dihydro-5,8-dimethyl-naphthalen-1-yl)-carbamic}

acid tert-butyl ester (3id): 99\% ee by HPLC analysis with a chiral column (Chiralcel OD, hexane/2-propanol $=90 / 10,0.5 \mathrm{~mL} / \mathrm{min}), \mathrm{t}_{\mathrm{R}}(R)-(-), 19.3 \mathrm{~min}$ (minor); $(S)-(+)$, 20.3 min (major). $[\alpha]^{25}+327.0\left(c 1.00, \mathrm{CHCl}_{3}\right)$ for 3id of $99 \%$ ee. mp $183-185{ }^{\circ} \mathrm{C}$; IR $\left(\mathrm{NaCl}, \mathrm{cm}^{-1}\right) 3195,2972,1701,1522,1448,1362,1247,1163,1114,999,862,753 .{ }^{1} \mathrm{H}$ NMR $\left(\mathrm{CDCl}_{3}\right) \delta 1.42(\mathrm{~s}, 9 \mathrm{H}), 2.29-2.38(\mathrm{~m}, 8 \mathrm{H}), 2.67-2.71(\mathrm{~m}, 2 \mathrm{H}), 3.36(\mathrm{~d}, J=6.0 \mathrm{~Hz}$, $1 \mathrm{H}), 3.58(\mathrm{t}, J=4.8 \mathrm{~Hz}, 4 \mathrm{H}), 4.40(\mathrm{~d}, J=6.0 \mathrm{~Hz}, 1 \mathrm{H}), 5.15(\mathrm{~d}, J=6.0 \mathrm{~Hz}, 1 \mathrm{H}), 5.93$ (dd, $J=9.9,6.0 \mathrm{~Hz}, 1 \mathrm{H}), 6.93(\mathrm{~d}, J=9.9 \mathrm{~Hz}, 1 \mathrm{H}), 6.99(\mathrm{~s}, 2 \mathrm{H}) ;{ }^{13} \mathrm{C} \mathrm{NMR}\left(\mathrm{CDCl}_{3}\right) \delta 155.4$, 
134.0, 132.7, 132.0, 130.4, 130.0, 127.2, 124.2, 79.6, 67.6, 63.1, 49.5, 44.2, 28.6, 19.1, 18.7. HRMS (EI) calcd for $\mathrm{C}_{21} \mathrm{H}_{30} \mathrm{~N}_{2} \mathrm{O}_{3}\left(\mathrm{M}^{+}\right)$: 358.2257 . Found: 358.2256 .

\section{(1S,2S)-(2-Dibenzylamino-1,2-dihydro-5,8-dimethyl-naphthalen-1-yl)-carbamic}

acid tert-butyl ester (3ie): $99 \%$ ee by HPLC analysis with a chiral column (Chiralcel $\mathrm{AD}$, hexane/2-propanol =99/1, $0.5 \mathrm{~mL} / \mathrm{min}), \mathrm{t}_{\mathrm{R}}(S)-(+), 13.3 \mathrm{~min}$ (major); $(R)-(-), 15.3$ min (minor). $[\alpha]_{\mathrm{D}}^{25}+175.8\left(c 1.00, \mathrm{CHCl}_{3}\right)$ for 3ie of $99 \%$ ee; IR $\left(\mathrm{NaCl}, \mathrm{cm}^{-1}\right) 3408$, 2974, 1707, 1493, 1365, 1228, 1163. ${ }^{1} \mathrm{H}$ NMR $\left(\mathrm{CDCl}_{3}\right) \delta 1.40(\mathrm{~s}, 9 \mathrm{H}), 2.29(\mathrm{~s}, 3 \mathrm{H})$, 2.38 (s, 3H), 3.39 (bs, 1H), 3.43 (d, $J=13.5 \mathrm{~Hz}, 2 \mathrm{H}), 3.53$ (d, $J=13.5 \mathrm{~Hz}, 2 \mathrm{H}), 4.43$ (d, $J=9.0 \mathrm{~Hz}, 1 \mathrm{H}), 5.32(\mathrm{~d}, J=9.0 \mathrm{~Hz}, 1 \mathrm{H}), 5.87(\mathrm{dd}, J=9.9,6.0 \mathrm{~Hz}, 1 \mathrm{H}), 6.88(\mathrm{~d}, J=9.9$ $\mathrm{Hz}, 1 \mathrm{H}), 6.95-7.01(\mathrm{~m}, 2 \mathrm{H}), 7.15-7.27(\mathrm{~m}, 6 \mathrm{H}), 7.35(\mathrm{~d}, J=6.9 \mathrm{~Hz}, 4 \mathrm{H}) ;{ }^{13} \mathrm{C} \mathrm{NMR}$ $\left(\mathrm{CDCl}_{3}\right) \delta 154.5,140.4,134.2,132.3,131.7,130.1,129.6,129.0,127.9,126.6,126.2$, 125.8, 79.2, 58.5, 53.5, 43.9, 28.3, 18.9, 18.6. HRMS (ESI) calcd for $\mathrm{C}_{31} \mathrm{H}_{37} \mathrm{~N}_{2} \mathrm{O}_{2}$ $\left[\mathrm{M}+\mathrm{H}^{+}\right]:$469.2849. Found: 469.2833 .

(1S,2S)-[2-( $N$-Methyl- $N$-phenyl-amino)-1,2-dihydro-5,8-dimethyl-naphthalen-1-y l]-carbamic acid tert-butyl ester (3if): $96 \%$ ee by HPLC analysis with a chiral column (Chiralcel OD-H, hexane/2-propanol =99/1, $1.0 \mathrm{~mL} / \mathrm{min}$ ), $\mathrm{t}_{\mathrm{R}}(S)-(+), 5.1 \mathrm{~min}$ (major); $(R)-(-), 5.6 \mathrm{~min}$ (minor). $[\alpha]_{\mathrm{D}}^{25}+292.6\left(c\right.$ 1.00, $\mathrm{CHCl}_{3}$ ) for 3if of $96 \%$ ee. mp 139-142 ${ }^{\circ} \mathrm{C}$; IR $\left(\mathrm{NaCl}, \mathrm{cm}^{-1}\right)$ 3402, 2975, 1715, 1597, 1504, 1365, 1165, 1107, 1034, 991, 900, 865, 812, 752, 693. ${ }^{1} \mathrm{H}$ NMR $\left(\mathrm{CDCl}_{3}\right) \delta 1.44(\mathrm{~s}, 9 \mathrm{H}), 2.26$ (s, 3H), 2.37 (s, 3H), 2.39 (s, $3 \mathrm{H}), 4.45(\mathrm{~d}, J=9.0 \mathrm{~Hz}, 1 \mathrm{H}), 4.55(\mathrm{~d}, J=9.0 \mathrm{~Hz}, 1 \mathrm{H}), 5.06(\mathrm{~d}, J=9.0 \mathrm{~Hz}, 1 \mathrm{H}), 5.93$ $(\mathrm{dd}, J=9.9,6.0 \mathrm{~Hz}, 1 \mathrm{H}), 6.76(\mathrm{t}, J=7.1 \mathrm{~Hz}, 1 \mathrm{H}), 6.97-7.58(\mathrm{~m}, 5 \mathrm{H}), 7.23-7.29(\mathrm{~m}, 2 \mathrm{H})$; ${ }^{13} \mathrm{C} \mathrm{NMR}\left(\mathrm{CDCl}_{3}\right) \delta 155.2,149.9,134.6,132.7,132.1,130.5,130.1,129.3,127.6,124.3$, 117.6, 114.4, 79.6, 57.8, 48.6, 32.4, 28.5, 19.1, 18.7. Anal. Calcd for $\mathrm{C}_{24} \mathrm{H}_{30} \mathrm{~N}_{2} \mathrm{O}_{2}: \mathrm{C}$, 76.16; H, 7.99; N, 7.40. Found: C, 75.88; H, 8.04; N, 7.35.

(1S,2S)-[2-(4-Phenyl-piperazin-1-yl)-1,2-dihydro-5,6,7,8-tetramethyl-naphthalen1-yl]-carbamic acid tert-butyl ester (3jc): $89 \%$ ee by HPLC analysis with a chiral column (Chiralcel AD, hexane/2-propanol = 90/10, 0.5 mL/min), $\mathrm{t}_{\mathrm{R}}(S)-(+), 11.2 \mathrm{~min}$ (major); $(R)-(-), 12.7$ min (minor). $[\alpha]^{25}+263.0\left(c 1.00, \mathrm{CHCl}_{3}\right)$ for $\mathbf{3 j c}$ of $89 \%$ ee; IR $\left(\mathrm{NaCl}, \mathrm{cm}^{-1}\right)$ 3242, 2973, 2842, 1700, 1598, 1494, 1449, 1364, 1233, 1167, 1046, 1002, 922, 871, 755, 692. ${ }^{1} \mathrm{H}$ NMR $\left(\mathrm{CDCl}_{3}\right) \delta 1.42(\mathrm{~s}, 9 \mathrm{H}), 2.24(\mathrm{~s}, 6 \mathrm{H}), 2.28(\mathrm{~s}, 3 \mathrm{H}), 2.30(\mathrm{~s}$, $3 \mathrm{H}), 2.43-2.57(\mathrm{~m}, 2 \mathrm{H}), 2.78-2.90(\mathrm{~m}, 2 \mathrm{H}), 3.07(\mathrm{t}, J=4.8 \mathrm{~Hz}, 4 \mathrm{H}), 3.44(\mathrm{~d}, J=6.0 \mathrm{~Hz}$, $1 \mathrm{H}), 4.42$ (d, $J=8.1 \mathrm{~Hz}, 1 \mathrm{H}), 5.24$ (d, $J=8.1 \mathrm{~Hz}, 1 \mathrm{H}), 5.92(\mathrm{dd}, J=9.9,6.0 \mathrm{~Hz}, 1 \mathrm{H})$, $6.79(\mathrm{t}, J=7.5 \mathrm{~Hz}, 1 \mathrm{H}), 6.84(\mathrm{~d}, J=8.1 \mathrm{~Hz}, 2 \mathrm{H}), 7.07(\mathrm{~d}, J=9.9 \mathrm{~Hz}, 1 \mathrm{H}), 7.19(\mathrm{~d}, J=$ $7.5 \mathrm{~Hz}, 1 \mathrm{H}), 7.22(\mathrm{~d}, J=7.5 \mathrm{~Hz}, 1 \mathrm{H}) ;{ }^{13} \mathrm{C} \mathrm{NMR}\left(\mathrm{CDCl}_{3}\right) \delta 155.1,151.6,135.9,135.1$, 
$132.2,130.5,130.3,129.2,128.1,128.0,123.3,119.6,116.1,79.4,62.8,49.6,49.0$, 44.7, 28.6, 17.2, 17.0, 15.5, 15.2. HRMS (EI) calcd for $\mathrm{C}_{29} \mathrm{H}_{39} \mathrm{~N}_{3} \mathrm{O}_{2}\left(\mathrm{M}^{+}\right)$: 461.3032 . Found: 461.3042 .

(1S,2S)-(2-Morpholin-4-yl-1,2-dihydro-5,6,7,8-tetramethyl-naphthalen-1-yl)-carb amic acid tert-butyl ester (3jd): $94 \%$ ee by HPLC analysis with a chiral column (Chiralpak AS, hexane/2-propanol = 98/2, $0.5 \mathrm{~mL} / \mathrm{min}$ ), $\mathrm{t}_{\mathrm{R}}(S)-(+), 17.5 \mathrm{~min}$ (major); $(R)-(-), 21.6 \mathrm{~min}$ (minor). $[\alpha]_{\mathrm{D}}^{25}+284.2\left(c\right.$ 1.00, $\left.\mathrm{CHCl}_{3}\right)$ for $\mathbf{3 j d}$ of $94 \%$ ee; $\mathrm{mp} 198-202$ ${ }^{\circ} \mathrm{C} ; \mathrm{IR}\left(\mathrm{NaCl}, \mathrm{cm}^{-1}\right)$ 3200, 2973, 2867, 1698, 1519, 1447, 1389, 1363, 1315, 1248, 1165 , 1117, 1047, 999, 861, 754. ${ }^{1} \mathrm{H}$ NMR $\left(\mathrm{CDCl}_{3}\right) \delta 1.41(\mathrm{~s}, 9 \mathrm{H}), 2.23(\mathrm{~s}, 3 \mathrm{H}), 2.25(\mathrm{~s}, 3 \mathrm{H})$, $2.28(\mathrm{~s}, 6 \mathrm{H}), 2.38-2.40(\mathrm{~m}, 2 \mathrm{H}), 2.61-2.74(\mathrm{~m}, 2 \mathrm{H}), 3.32(\mathrm{~d}, J=6.0 \mathrm{~Hz}, 1 \mathrm{H}), 3.57(\mathrm{t}, J=$ $4.8 \mathrm{~Hz}, 4 \mathrm{H}), 4.40(\mathrm{~d}, J=8.1 \mathrm{~Hz}, 1 \mathrm{H}), 5.20$ (d, $J=8.1 \mathrm{~Hz}, 1 \mathrm{H}), 5.88(\mathrm{dd}, J=9.9,6.0 \mathrm{~Hz}$, $1 \mathrm{H}), 7.04(\mathrm{~d}, J=9.9 \mathrm{~Hz}, 1 \mathrm{H}) ;{ }^{13} \mathrm{C}$ NMR $\left(\mathrm{CDCl}_{3}\right) \delta 155.1,136.0,135.2,132.2,130.5$, $130.3,128.2$, 128.0, 123.1, 79.5, 67.6, 63.2, 49.7, 44.9, 28.6, 17.3, 17.0, 15.5, 15.2 . HRMS (EI) calcd for $\mathrm{C}_{23} \mathrm{H}_{34} \mathrm{~N}_{2} \mathrm{O}_{3}\left(\mathrm{M}^{+}\right)$: 386.2573 . Found: 386.2569 .

(1S,2S)-(2-N,N-Dibenzylamino-1,2-dihydro-5,6,7,8-tetramethyl-naphthalen-1-yl)carbamic acid tert-butyl ester (3je): 99\% ee by HPLC analysis with a chiral column (Chiralcel AD, hexane/2-propanol $=99 / 1,0.5 \mathrm{~mL} / \mathrm{min}), \mathrm{t}_{\mathrm{R}}(S)-(+), 9.6 \mathrm{~min}$ (major); $(R)-(-), 12.0$ min (minor). $[\alpha]^{25}+265.8\left(c\right.$ 1.00, $\left.\mathrm{CHCl}_{3}\right)$ for 3 je of $99 \%$ ee; IR $(\mathrm{NaCl}$, $\left.\mathrm{cm}^{-1}\right) 3418,2925,2358,1705,1489,1365,1165,1047 .{ }^{1} \mathrm{H} \mathrm{NMR}\left(\mathrm{CDCl}_{3}\right) \delta 1.39(\mathrm{~s}, 9 \mathrm{H})$, $2.21(\mathrm{~s}, 3 \mathrm{H}), 2.25(\mathrm{~s}, 6 \mathrm{H}), 2.33(\mathrm{~s}, 3 \mathrm{H}), 3.38(\mathrm{~d}, J=6.0 \mathrm{~Hz}, 1 \mathrm{H}), 3.44(\mathrm{~d}, J=13.5 \mathrm{~Hz}$, 2H), $3.52(\mathrm{~d}, J=13.5 \mathrm{~Hz}, 2 \mathrm{H}), 4.42(\mathrm{~d}, J=9.0 \mathrm{~Hz}, 1 \mathrm{H}), 5.38(\mathrm{~d}, J=9.0 \mathrm{~Hz}, 1 \mathrm{H}), 5.83$ $(\mathrm{dd}, J=9.9,6.0 \mathrm{~Hz}, 1 \mathrm{H}), 7.01(\mathrm{~d}, J=9.9 \mathrm{~Hz}, 1 \mathrm{H}), 7.14-7.25(\mathrm{~m}, 6 \mathrm{H}), 7.35(\mathrm{~d}, J=7.2$ $\mathrm{Hz}, 4 \mathrm{H}) ;{ }^{13} \mathrm{C} \mathrm{NMR}\left(\mathrm{CDCl}_{3}\right) \delta 154.7,140.7,135.8,135.0,132.5,130.4,130.2,129.2$, $128.1,127.9,127.3,126.7,124.8,79.2,58.6,53.7,44.9,28.5,17.2,17.0,15.4,15.2$. HRMS (ESI) calcd for $\mathrm{C}_{33} \mathrm{H}_{41} \mathrm{~N}_{2} \mathrm{O}_{2}\left[\mathrm{M}+\mathrm{H}^{+}\right]$: 497.3162 . Found: 497.3174 .

(1S,2S)-[2-(N-Phenyl-piperazin-1-yl)-1,2-dihydro-6,7-dimethyl-naphthalen-1-yl]carbamic acid tert-butyl ester (3kc): $94 \%$ ee by HPLC analysis with a chiral column (Chiralcel AD, hexane/2-propanol =90/10, $0.5 \mathrm{~mL} / \mathrm{min}$ ), $\mathrm{t}_{\mathrm{R}}(S)-(+), 17.9 \mathrm{~min}$ (major); $(R)-(-), 19.6$ min (minor). $[\alpha]_{\mathrm{D}}^{25}+304.8$ (c 1.00, $\left.\mathrm{CHCl}_{3}\right)$ for $3 \mathbf{k c}$ of $94 \%$ ee. $\mathrm{mp}$ $178-181{ }^{\circ} \mathrm{C}$; IR $\left(\mathrm{NaCl}, \mathrm{cm}^{-1}\right)$ 3320, 2971, 2855, 1698, 1504, 1450, 1390, 1365, 1289 , 1248, 1169, 1115, 1049, 1003, 882, 754. ${ }^{1} \mathrm{H}$ NMR $\left(\mathrm{CDCl}_{3}\right) \delta 1.45(\mathrm{~s}, 9 \mathrm{H}), 2.24(\mathrm{~s}, 3 \mathrm{H})$, $2.25(\mathrm{~s}, 3 \mathrm{H}), 2.62-2.69(\mathrm{~m}, 2 \mathrm{H}), 2.77-2.84(\mathrm{~m}, 2 \mathrm{H}), 3.10(\mathrm{t}, J=4.8 \mathrm{~Hz}, 4 \mathrm{H}), 3.43(\mathrm{t}, J=$ $6.0 \mathrm{~Hz}, 1 \mathrm{H}), 4.44-4.58(\mathrm{~m}, 1 \mathrm{H}), 4.92-5.05(\mathrm{~m}, 1 \mathrm{H}), 5.91$ (dd, $J=9.9,6.0 \mathrm{~Hz}, 1 \mathrm{H}), 6.62$ $(\mathrm{d}, J=9.9 \mathrm{~Hz}, 1 \mathrm{H}), 6.82(\mathrm{t}, J=7.5 \mathrm{~Hz}, 1 \mathrm{H}), 6.86(\mathrm{~d}, J=8.1 \mathrm{~Hz}, 2 \mathrm{H}), 6.89(\mathrm{~s}, 1 \mathrm{H}), 7.11$ 
(s, 1H), $7.21(\mathrm{~d}, J=7.5 \mathrm{~Hz}, 1 \mathrm{H}), 7.24(\mathrm{~d}, J=7.5 \mathrm{~Hz}, 1 \mathrm{H}) ;{ }^{13} \mathrm{C} \mathrm{NMR}\left(\mathrm{CDCl}_{3}\right) \delta 155.3$, 151.6, 136.9, 136.4, 132.7, 130.2, 129.7, 129.5, 129.2, 128.3, 124.3, 119.6, 116.2, 79.5, 64.0, 49.7, 48.8, 48.1, 28.6, 19.8, 19.6. HRMS (EI) calcd for $\mathrm{C}_{27} \mathrm{H}_{35} \mathrm{~N}_{3} \mathrm{O}_{2}\left(\mathrm{M}^{+}\right)$: 443.2720. Found: 443.2729.

\section{(1S,2S)-(2-Morpholin-4-yl-1,2-dihydro-6,7-dimethyl-naphthalen-1-yl)-carbamic}

acid tert-butyl ester (3kd): $>99 \%$ ee by HPLC analysis with a chiral column (Chiralcel $\mathrm{AS}$, hexane/2-propanol =98/2, $0.5 \mathrm{~mL} / \mathrm{min}$ ), $\mathrm{t}_{\mathrm{R}}(S)-(+), 29.4 \mathrm{~min}$ (major); $(R)-(-), 34.9$ $\min$ (minor). $[\alpha]_{\mathrm{D}}^{25}+251.4\left(c 1.00, \mathrm{CHCl}_{3}\right.$ ) for $3 \mathbf{k d}$ of $>99 \%$ ee; $\mathrm{mp} 57-60{ }^{\circ} \mathrm{C}$; IR $(\mathrm{NaCl}$, $\mathrm{cm}^{-1}$ ) 3320, 2971, 2855, 1698, 1504, 1450, 1390, 1365, 1289, 1248, 1169, 1157, 1049, 1103, 883, 754. ${ }^{1} \mathrm{H}$ NMR $\left(\mathrm{CDCl}_{3}\right) \delta 1.45(\mathrm{~s}, 9 \mathrm{H}), 2.23(\mathrm{~s}, 3 \mathrm{H}), 2.25(\mathrm{~s}, 3 \mathrm{H}), 2.44-2.53$ $(\mathrm{m}, 2 \mathrm{H}), 2.61-2.68(\mathrm{~m}, 2 \mathrm{H}), 3.32(\mathrm{t}, J=6.0 \mathrm{~Hz}, 1 \mathrm{H}), 3.61(\mathrm{t}, J=4.8 \mathrm{~Hz}, 4 \mathrm{H}), 4.43-4.58$ $(\mathrm{m}, 1 \mathrm{H}), 4.87-5.02(\mathrm{~m}, 1 \mathrm{H}), 5.87(\mathrm{dd}, J=9.9,6.0 \mathrm{~Hz}, 1 \mathrm{H}), 6.60(\mathrm{~d}, J=9.9 \mathrm{~Hz}, 1 \mathrm{H})$, $6.87(\mathrm{~s}, 1 \mathrm{H}), 7.11(\mathrm{~s}, 1 \mathrm{H}) ;{ }^{13} \mathrm{C} \mathrm{NMR}\left(\mathrm{CDCl}_{3}\right) \delta 155.4,137.0,136.6,132.7,130.2,129.8$, 129.5, 128.3, 124.1, 79.6, 67.6, 64.5, 49.4, 48.3, 28.7, 19.8, 19.6. Anal. Calcd for $\mathrm{C}_{21} \mathrm{H}_{30} \mathrm{~N}_{2} \mathrm{O}_{3}$ : C, 70.36; H, 8.44; N, 7.81. Found: C, 70.06; H, 8.79; N, 7.71.

\section{(1S,2S)-(2-Dibenzylamino-1,2-dihydro-6,7-dimethyl-naphthalen-1-yl)-carbamic}

acid tert-butyl ester (3ke): $97 \%$ ee by HPLC analysis with a chiral column (Chiralcel $\mathrm{OD}$, hexane/2-propanol =99/1, $0.5 \mathrm{~mL} / \mathrm{min}$ ), $\mathrm{t}_{\mathrm{R}}(S)-(+), 14.2 \mathrm{~min}$ (major); $(R)-(-), 18.7$ $\min$ (minor). $[\alpha]^{25}+135.8$ (c 1.00, $\mathrm{CHCl}_{3}$ ) for 3ke of $97 \%$ ee; IR $\left(\mathrm{NaCl}, \mathrm{cm}^{-1}\right) 3415$, 2973, 1713, 1494, 1364, 1245, 1166. ${ }^{1} \mathrm{H}$ NMR $\left(\mathrm{CDCl}_{3}\right) \delta 1.51(\mathrm{~s}, 9 \mathrm{H}), 2.19(\mathrm{~s}, 3 \mathrm{H})$, $2.23(\mathrm{~s}, 3 \mathrm{H}), 3.41(\mathrm{bs}, 1 \mathrm{H}), 3.53(\mathrm{~d}, J=13.5 \mathrm{~Hz}, 2 \mathrm{H}), 3.70(\mathrm{~d}, J=13.5 \mathrm{~Hz}, 2 \mathrm{H})$, 4.43-4.46 (m, 1H), $5.18(\mathrm{~m}, 1 \mathrm{H}), 5.93-5.96(\mathrm{~m}, 1 \mathrm{H}), 6,54(\mathrm{dd}, J=9.9,1.8 \mathrm{~Hz}, 1 \mathrm{H}), 6.82$ $(\mathrm{s}, 1 \mathrm{H}), 7.08(\mathrm{~s}, 1 \mathrm{H}), 7.15-7.28(\mathrm{~m}, 6 \mathrm{H}), 7.37(\mathrm{~d}, J=6.9 \mathrm{~Hz}, 4 \mathrm{H}) ;{ }^{13} \mathrm{C} \mathrm{NMR}\left(\mathrm{CDCl}_{3}\right) \delta$ 155.6, 140.3, 136.6, 136.1, 133.1, 130.3, 129.4, 129.3, 128.9, 128.3, 128.1, 127.0, 125.8, 79.5, 59.5, 54.0, 48.9, 28.7, 19.9, 19.5. HRMS (ESI) calcd for $\mathrm{C}_{31} \mathrm{H}_{37} \mathrm{~N}_{2} \mathrm{O}_{2}\left[\mathrm{M}+\mathrm{H}^{+}\right]$: 469.2849. Found: 469.2849 .

(1S,2S)-(6,7-Dimethoxy-2-pyrrolidin-1-yl-1,2-dihydro-naphthalen-1-yl)-carbamic acid tert- butyl ester (3la): $>99 \%$ ee by HPLC analysis with a chiral column (Chiralcel $\mathrm{OD}$, hexane/2-propanol =90/10, $1.0 \mathrm{~mL} / \mathrm{min}), \mathrm{t}_{\mathrm{R}}(R)-(-), 6.0 \mathrm{~min}$ (minor); $(S)-(+), 7.9$ $\min$ (major). $[\alpha]_{\mathrm{D}}^{25}+152.7$ (c 1.00, $\mathrm{CHCl}_{3}$ ) for 3la of $>99 \%$ ee. IR $\left(\mathrm{NaCl}, \mathrm{cm}^{-1}\right) 3361$, 2967, 2253, 1705, 1605, 1515, 1267, 1169, 732. ${ }^{1} \mathrm{H}$ NMR $\left(\mathrm{CDCl}_{3}\right) \delta 1.43(\mathrm{~s}, 9 \mathrm{H})$, $1.69-1.71(\mathrm{~m}, 4 \mathrm{H}), 2.58-2.60(\mathrm{~m}, 2 \mathrm{H}), 2.70-2.72(\mathrm{~m}, 2 \mathrm{H}), 3.26(\mathrm{bs}, 1 \mathrm{H}), 3.86(\mathrm{~s}, 3 \mathrm{H})$, $3.88(\mathrm{~s}, 3 \mathrm{H}), 4.70(\mathrm{~d}, J=8.0 \mathrm{~Hz}, 1 \mathrm{H}), 4.86(\mathrm{~d}, J=8.0 \mathrm{~Hz}, 1 \mathrm{H}), 5.88(\mathrm{dd}, J=9.9,6.0 \mathrm{~Hz}$, $1 \mathrm{H}), 6.54(\mathrm{~d}, J=9.9 \mathrm{~Hz}, 1 \mathrm{H}), 6.63(\mathrm{~s}, 1 \mathrm{H}), 6.88(\mathrm{~s}, 1 \mathrm{H}) ;{ }^{13} \mathrm{C} \mathrm{NMR}\left(\mathrm{CDCl}_{3}\right) \delta 155.2$, 
$148.7,148.6,128.8,127.3,125.1,123.9,112.1,110.2,79.5,61.5,56.1,56.0,50.5,28.5$, 23.5. HRMS (EI) calcd for $\mathrm{C}_{22} \mathrm{H}_{26} \mathrm{~N}_{2} \mathrm{O}_{2} \mathrm{~S}\left(\mathrm{M}^{+}\right)$: 376.2362. Found: 376.2371 .

\section{(1S,2S)-(2-Morpholin-4-yl-1,2-dihydro-6,7-dimethoxynaphthalen-1-yl)-carbamic}

acid tert-butyl ester (3ld): $96 \%$ ee by HPLC analysis with a chiral column (Chiralcel $\mathrm{AD}$, hexane/2-propanol = 90/10, $0.5 \mathrm{~mL} / \mathrm{min}$ ), $\mathrm{t}_{\mathrm{R}}(S)-(+), 26.9 \mathrm{~min}$ (major); $(R)-(-), 31.9$ min (minor). $[\alpha]^{25}{ }_{\mathrm{D}}+280.2\left(c 1.00, \mathrm{CHCl}_{3}\right)$ for 3ld of $96 \%$ ee. IR $\left(\mathrm{NaCl}, \mathrm{cm}^{-1}\right) 3348$, 2961, 2855, 1698, 1604, 1514, 1463, 1365, 1316, 1266, 1170, 1115, 1001, 916, 869, 768, 731, 646. ${ }^{1} \mathrm{H}$ NMR $\left(\mathrm{CDCl}_{3}\right) \delta 1.45(\mathrm{~s}, 9 \mathrm{H}), 2.42-2.50(\mathrm{~m}, 2 \mathrm{H}), 2.63-2.70(\mathrm{~m}, 2 \mathrm{H})$, 3.29-3.31 (m, 1H), $3.61(\mathrm{t}, J=4.8 \mathrm{~Hz}, 4 \mathrm{H}), 3.88(\mathrm{~s}, 3 \mathrm{H}), 3.90(\mathrm{~s}, 3 \mathrm{H}), 4.56(\mathrm{~d}, J=8.1$ $\mathrm{Hz}, 1 \mathrm{H}), 4.93(\mathrm{~d}, J=8.1 \mathrm{~Hz}, 1 \mathrm{H}), 5.84(\mathrm{dd}, J=9.9,6.0 \mathrm{~Hz}, 1 \mathrm{H}), 6.59$ (d, $J=9.9 \mathrm{~Hz}$, $1 \mathrm{H}), 6.62(\mathrm{~s}, 1 \mathrm{H}), 6.88(\mathrm{~s}, 1 \mathrm{H}) ;{ }^{13} \mathrm{C} \mathrm{NMR}\left(\mathrm{CDCl}_{3}\right) \delta 155.3,149.0,148.8,129.5,127.7$, $125.4,122.9,111.5,110.2,79.7,67.6,64.1,56.3,56.1,49.3,48.2$, 28.6. HRMS (EI) calcd for $\mathrm{C}_{21} \mathrm{H}_{30} \mathrm{~N}_{2} \mathrm{O}_{5}\left(\mathrm{M}^{+}\right)$: 390.2165 . Found: 390.2154 .

(1S,2S)-[2-( $N$-Methyl- $N$-phenylamino)-1,2-dihydro-6,7-dimethoxy-naphthalen-1yl]-carbamic acid tert-butyl ester (3lf): $99 \%$ ee by HPLC analysis with a chiral column (Chiralcel AD, hexane/2-propanol =90/10, $0.5 \mathrm{~mL} / \mathrm{min}), \mathrm{t}_{\mathrm{R}}(S)-(+), 24.9 \mathrm{~min}$ (major); $(R)-(-), 28.6$ min (minor). $[\alpha]^{25}+91.8\left(c 1.00, \mathrm{CHCl}_{3}\right)$ for 3lf of $99 \%$ ee. $\mathrm{mp}$ 159-161 ${ }^{\circ} \mathrm{C}$; IR $\left(\mathrm{NaCl}, \mathrm{cm}^{-1}\right)$ 3363, 2975, 1711, 1598, 1505, 1364, 1273, 1228, 1164 , 1108, 1013, 944, 868, 749, 692. ${ }^{1} \mathrm{H}$ NMR $\left(\mathrm{CDCl}_{3}\right) \delta 1.37$ (s, 9H), 2.75 (s, 3H), 3.87 (s, $3 \mathrm{H}), 3.89(\mathrm{~s}, 3 \mathrm{H}), 4.52(\mathrm{~d}, J=9.0 \mathrm{~Hz}, 1 \mathrm{H}), 4.69(\mathrm{~d}, J=9.0 \mathrm{~Hz}, 1 \mathrm{H}), 5.06(\mathrm{t}, J=9.0 \mathrm{~Hz}$, $1 \mathrm{H}), 5.82(\mathrm{dd}, J=9.9,6.0 \mathrm{~Hz}, 1 \mathrm{H}), 6.56(\mathrm{dd}, J=9.9,1.8 \mathrm{~Hz}, 1 \mathrm{H}), 6.66(\mathrm{~s}, 3 \mathrm{H})$, 6.86-6.89 (m, 3H), $7.23(\mathrm{t}, J=7.2 \mathrm{~Hz}, 2 \mathrm{H}) ;{ }^{13} \mathrm{C} \mathrm{NMR}\left(\mathrm{CDCl}_{3}\right) \delta 155.6,150.1,148.8$, $148.6,129.8,129.4,128.5,126.9,125.7,117.3,113.8,110.5,79.7,60.4,56.3,56.2$, 52.2, 33.1, 28.5. Anal. Calcd for $\mathrm{C}_{24} \mathrm{H}_{30} \mathrm{~N}_{2} \mathrm{O}_{4}$ : C, 70.22; H, 7.37; N, 6.82. Found: C, $69.82 ; \mathrm{H}, 7.07 ; \mathrm{N}, 6.73$.

\section{(1S,2S)-(6,7-Difluoro-2-pyrrolidin-1-yl-1,2-dihydro-naphthalen-1-yl)-carbamic}

acid tert-butyl ester (3ma): $>99 \%$ ee by HPLC analysis with a chiral column (Chiralcel OD-H, hexane/2-propanol =90/10, $1.0 \mathrm{~mL} / \mathrm{min}), \mathrm{t}_{\mathrm{R}}(S)-(+), 7.5 \mathrm{~min}$ (major); $(R)-(-)$, 9.6 min (minor). $[\alpha]^{25}+272.6\left(c 1.00, \mathrm{CHCl}_{3}\right)$ for $\mathbf{3 m a}$ of $>99 \%$ ee. IR $\left(\mathrm{NaCl}, \mathrm{cm}^{-1}\right)$ $3343,2970,1696,1505,1366,1310,1250,1169,1054,1023,912,882,742 .{ }^{1} \mathrm{H}$ NMR $\left(\mathrm{CDCl}_{3}\right) \delta 1.44(\mathrm{~s}, 9 \mathrm{H}), 1.69-1.72(\mathrm{~m}, 4 \mathrm{H}), 2.58-2.68(\mathrm{~m}, 4 \mathrm{H}), 3.29(\mathrm{t}, J=6.0 \mathrm{~Hz}, 1 \mathrm{H})$, 4.70 (bs, 1H), 4.86 (bs, 1H), 6.03 (dd, $J=9.9,6.0 \mathrm{~Hz}, 1 \mathrm{H}), 6.51$ (d, $J=9.9 \mathrm{~Hz}, 1 \mathrm{H})$, $6.90(\mathrm{dd}, J=10.5,7.8 \mathrm{HZ}, 1 \mathrm{H}), 7.18(\mathrm{dd}, J=10.5,7.8,1 \mathrm{H}) ;{ }^{13} \mathrm{C} \mathrm{NMR}\left(\mathrm{CDCl}_{3}\right) \delta 155.3$, $151.0(\mathrm{q}, J=13.0 \mathrm{~Hz}), 148.6(\mathrm{q}, J=13.0 \mathrm{~Hz}), 132.1(\mathrm{dd}, J=4.0,1.2 \mathrm{~Hz}), 129.1(\mathrm{dd}, J=$ 
6.3, 4.0 Hz), 127.6, 127.3, $117.6(\mathrm{~d}, J=18.2 \mathrm{~Hz}), 115.5(\mathrm{~d}, J=18.2 \mathrm{~Hz}), 79.9,61.1$, 50.4, 49.9, 28.5, 23.6.; ${ }^{19} \mathrm{~F} \mathrm{NMR}\left(\mathrm{CDCl}_{3}\right) \delta-138.1$ (t, $\left.J=9.0 \mathrm{~Hz}\right),-139.5(\mathrm{t}, J=9.0 \mathrm{~Hz})$. HRMS (EI) calcd for $\mathrm{C}_{19} \mathrm{H}_{24} \mathrm{~N}_{2} \mathrm{O}_{2} \mathrm{~F}_{2}\left(\mathrm{M}^{+}\right)$: 351.1884. Found: 351.1887.

\section{(1S,2S)-(2-Piperidin-1-yl-1,2-dihydro-5,8-dimethyl-naphthalen-1-yl)-carbamic}

acid tert-butyl ester (3mb): 97\% ee by HPLC analysis with a chiral column (Chiralcel OD-H, hexane/2-propanol = 90/10, $0.5 \mathrm{~mL} / \mathrm{min}), \mathrm{t}_{\mathrm{R}}(S)-(+), 15.0 \mathrm{~min}(\operatorname{minor}) ;(R)-(-)$, 16.4 min (major). $[\alpha]^{25}+236.2\left(c 1.00, \mathrm{CHCl}_{3}\right.$ ) for $\mathbf{3 m b}$ of $97 \%$ ee. $\mathrm{mp} 112-115{ }^{\circ} \mathrm{C}$; IR $\left(\mathrm{NaCl}, \mathrm{cm}^{-1}\right)$ 3266, 2933, 1679, 1598, 1536, 1505, 1366, 1303, 1277, 1173, 1119, 880, 778. ${ }^{1} \mathrm{H}$ NMR $\left(\mathrm{CDCl}_{3}\right) \delta$ 1.34-1.56 (m, 15H), 2.41-2.57 (m, 4H), 3.31-3.34 (m, 1H), 4.51-4.70 (m, 1H), 4.85-5.02 (m, 1H), $6.02(\mathrm{dd}, J=9.9,6.0 \mathrm{~Hz}, 1 \mathrm{H}), 6.50(\mathrm{dd}, J=9.9$, $1.5 \mathrm{~Hz}, 1 \mathrm{H}), 6.88(\mathrm{dd}, J=10.5,7.8 \mathrm{~Hz}, 1 \mathrm{H}), 7.20(\mathrm{dd}, J=10.5,7.8 \mathrm{~Hz}, 1 \mathrm{H}) ;{ }^{13} \mathrm{C} \mathrm{NMR}$ $\left(\mathrm{CDCl}_{3}\right) \delta 155.17,151.39(\mathrm{q}, J=13.4 \mathrm{~Hz}), 148.10(\mathrm{q}, J=13.4 \mathrm{~Hz}), 133.07(\mathrm{t}, J=4.5$ $\mathrm{Hz}), 129.46$ (dd, $J=6.3,4.5 \mathrm{~Hz}), 127.53$ (d, $J=4.5 \mathrm{~Hz}), 127.50$ (d, $J=4.5 \mathrm{~Hz}), 116.77$ $(\mathrm{d}, J=18.0 \mathrm{~Hz}), 115.33(\mathrm{~d}, J=18.0 \mathrm{~Hz}), 79.96,64.80,50.24,48.19,28.59,26.61$, 24.74.; ${ }^{19} \mathrm{~F} \mathrm{NMR}\left(\mathrm{CDCl}_{3}\right) \delta-138.2(\mathrm{t}, J=10.0 \mathrm{~Hz}),-139.9(\mathrm{t}, J=10.0 \mathrm{~Hz})$. HRMS (ESI) calcd for $\mathrm{C}_{20} \mathrm{H}_{27} \mathrm{~N}_{2} \mathrm{O}_{2} \mathrm{~F}_{2}\left[\mathrm{M}+\mathrm{H}^{+}\right]$: 365.2035. Found: 365.2052.

\section{(1S,2S)-[2-(N-Methyl- $N$-phenylamino)-1,2-dihydro-6,7-difluoro-naphthalen-1-yl]} -carbamic acid tert-butyl ester (3mf): 98\% ee by HPLC analysis with a chiral column (Chiralcel OD-H, hexane/2-propanol = 99/1, $1.0 \mathrm{~mL} / \mathrm{min}$ ), $\mathrm{t}_{\mathrm{R}}(R)-(-), 10.0 \mathrm{~min}$ (minor); $(S)-(+), 13.7$ min (major). $[\alpha]_{\mathrm{D}}^{25}+16.8\left(c 1.00, \mathrm{CHCl}_{3}\right)$ for $\mathbf{3 m f}$ of $98 \%$ ee. $\mathrm{mp} 148-151$ ${ }^{\circ} \mathrm{C}$; IR $\left(\mathrm{NaCl}, \mathrm{cm}^{-1}\right)$ 3342, 2976, 1680, 1598, 1503, 1367, 1305, 1169, 1104, 940, 885, 779, 745. ${ }^{1} \mathrm{H}$ NMR $\left(\mathrm{CDCl}_{3}\right) \delta 1.34(\mathrm{~s}, 9 \mathrm{H}), 2.84(\mathrm{~s}, 3 \mathrm{H}), 4.46(\mathrm{~d}, J=9.9 \mathrm{~Hz}, 1 \mathrm{H}), 4.78$ $(\mathrm{d}, J=10.5 \mathrm{~Hz}, 1 \mathrm{H}), 5.14(\mathrm{~d}, J=9.9 \mathrm{~Hz}, 1 \mathrm{H}), 5.97$ (dd, $J=9.9,2.4 \mathrm{~Hz}, 1 \mathrm{H}), 6.49$ (dd, $J$ $=9.9,2.4 \mathrm{~Hz}, 1 \mathrm{H}), 6.75(\mathrm{t}, J=7.2 \mathrm{~Hz}, 1 \mathrm{H}), 6.83(\mathrm{~d}, J=8.5 \mathrm{~Hz}, 2 \mathrm{H}), 6.92(\mathrm{dd}, J=10.5$, $7.8 \mathrm{~Hz}, 1 \mathrm{H}), 7.16(\mathrm{dd}, J=10.5,7.8 \mathrm{~Hz}, 1 \mathrm{H}), 7.23(\mathrm{dd}, J=8.5,7.2 \mathrm{~Hz}, 2 \mathrm{H}) ;{ }^{13} \mathrm{C} \mathrm{NMR}$ $\left(\mathrm{CDCl}_{3}\right) \delta 155.51,151.40(\mathrm{t}, \mathrm{J}=13.0 \mathrm{~Hz}), 150.18,148.10(\mathrm{t}, J=13.0 \mathrm{~Hz}), 133.34$, 131.33, 129.76, 129.45, 128.45, 117.67, 115.65 (d, $J=18.0 \mathrm{~Hz}), 113.99,80.14,60.81$, 52.09, 33.40, 28.40.; ${ }^{19} \mathrm{~F}$ NMR $\left(\mathrm{CDCl}_{3}\right) \delta-138.0(\mathrm{t}, J=11.1 \mathrm{~Hz}),-140.2(\mathrm{t}, J=11.1$ $\mathrm{Hz}$ ). HRMS (EI) calcd for $\mathrm{C}_{22} \mathrm{H}_{24} \mathrm{~N}_{2} \mathrm{O}_{2} \mathrm{~F}_{2}\left(\mathrm{M}^{+}\right)$: 386.1806 . Found: 386.1805 .

\section{Preparation of dialkylammonium iodide from the corresponding chloride.} Ammonium chloride was dissolved in appropriate solvent $(\mathrm{EtOH}$ in the case of diethylammonium chloride and $\mathrm{MeOH}$ in dimethylammonium chloride) (0.5-1.0 M). $\mathrm{NaI}$ ( 1 equiv.) solution $(0.5-1.0 \mathrm{M})$ in the same solvent was added dropwise to the chloride solution at room temperature. White precipitate was immediately formed. After 
addition was completed, the mixture was stirred for $0.5-1 \mathrm{~h}$. After filtration to remove $\mathrm{NaCl}$, the solution was evaporated. The residue was dissolved in $\mathrm{CH}_{2} \mathrm{Cl}_{2}$ and insoluble precipitate was filtered off again. Evaporation of the solvent gave the corresponding iodide (quantitative yield at this stage). If product had yellow color, the solid was carefully washed with the cold solvent in which the reaction was carried out.

Catalytic Ring-Opening of $1 \mathrm{a}$ with $\mathrm{Et}_{2} \mathrm{NH}_{2} \mathrm{I}$ in the presence of $i$-Pr $2 \mathrm{NEt}$ in dioxane. To a stirred solution of $1 \mathbf{a}(100 \mathrm{mg}, 0.41 \mathrm{mmol}),[\mathrm{Rh}(\mathrm{cod}) \mathrm{Cl}]_{2}(2 \mathrm{mg}, 4 \mu \mathrm{mol})$, dppf ( $7 \mathrm{mg}, 12 \mu \mathrm{mol})$, and $\mathrm{Et}_{2} \mathrm{NH}_{2} \mathrm{I}(165 \mathrm{mg}, 0.82 \mathrm{mmol})$ in dry 1,4-dioxane $(2.0 \mathrm{~mL})$ was added $i$ - $\operatorname{Pr}_{2} \mathrm{NEt}(0.2 \mathrm{~mL}, 1.23 \mathrm{mmol})$ under nitrogen atmosphere. The reaction mixture was allowed to stir at $110{ }^{\circ} \mathrm{C}$ for $14 \mathrm{~h}$. The resulting mixture was cooled to room temperature and quenched with $1 \mathrm{~N} \mathrm{NaOH}$ solution. The mixture was extracted with $\mathrm{CH}_{2} \mathrm{Cl}_{2}$ and the separated organic layer was dried over anhydrous $\mathrm{MgSO}_{4}$. After evaporation under reduced pressure, the residue was purified by flash column chromatography (hexane/ethyl acetate $=70 / 30)$ to give $115 \mathrm{mg}(90 \%$ yield) of 3ak. Similarly, the ring-opening product 3al (89\% yield) was prepared under the same condition.

\section{(1S,2S)-(2-Diethylamino-1,2-dihydro-naphthalen-1-yl)-carbamic acid tert-butyl} ester (3ak): $85 \%$ ee by HPLC analysis with a chiral column (Chiralcel AD, hexane/2-propanol =90/10, $0.5 \mathrm{~mL} / \mathrm{min}), \mathrm{t}_{\mathrm{R}}(S)-(+), 10.4 \mathrm{~min}$ (major); $(R)-(-), 11.4 \mathrm{~min}$ (minor). $[\alpha]^{25}+145.4\left(c 1.00, \mathrm{CHCl}_{3}\right)$ for 3ak of $85 \%$ ee; mp $85-87{ }^{\circ} \mathrm{C}$; IR $(\mathrm{NaCl}$, $\mathrm{cm}^{-1}$ ) 3346, 1701, 1524, 1488, 1452, 1390, 1305, 1250, 1171, 1113, 1045, 1023, 979, 903, 858, 780. ${ }^{1} \mathrm{H}$ NMR $\left(\mathrm{CDCl}_{3}\right) \delta 1.01(\mathrm{t}, J=6.6 \mathrm{~Hz}, 6 \mathrm{H}), 1.46(\mathrm{~s}, 9 \mathrm{H}), 2.47-2.60(\mathrm{~m}$, $4 \mathrm{H}), 3.51-3.55(\mathrm{~m}, 1 \mathrm{H}), 4.63(\mathrm{bs}, 1 \mathrm{H}), 4.93(\mathrm{t}, J=6.6 \mathrm{~Hz}, 1 \mathrm{H}), 5.97(\mathrm{dd}, J=9.9,4.8 \mathrm{~Hz}$, $1 \mathrm{H}), 6.58(\mathrm{dd}, J=9.9,1.5 \mathrm{~Hz}, 1 \mathrm{H}), 7.05-7.08(\mathrm{~m}, 1 \mathrm{H}), 7.19-7.24(\mathrm{~m}, 2 \mathrm{H}), 7.32-7.35(\mathrm{~m}$, $1 \mathrm{H}) ;{ }^{13} \mathrm{C} \mathrm{NMR}\left(\mathrm{CDCl}_{3}\right) \delta 155.4,135.7,132.5,129.0,127.9,127.8,127.7,127.4,126.4$, 79.2, 60.5, 49.6, 43.8, 23.4, 14.2. Anal. Calcd for $\mathrm{C}_{19} \mathrm{H}_{29} \mathrm{~N}_{2} \mathrm{O}_{2}: \mathrm{C}, 72.12 ; \mathrm{H}, 8.92 ; \mathrm{N}$, 8.85.Found: C, 71.90; H, 8.90; N, 8.80.

\section{Asymmetric Ring-Opening of 1a with $\mathrm{Me}_{2} \mathrm{NH}_{2} \mathrm{Cl}$ in the presence of $\mathrm{Et}_{3} \mathrm{~N}$ in} tetrahydropyran. To a stirred solution of $1 \mathbf{a}(100 \mathrm{mg}, 0.41 \mathrm{mmol}),[\mathrm{Rh}(\mathrm{cod}) \mathrm{Cl}]_{2}(5 \mathrm{mg}$, $10 \mu \mathrm{mol}), 4 \mathrm{a}(32 \mathrm{mg}, 45 \mu \mathrm{mol})$, and $\mathrm{Me}_{2} \mathrm{NH}_{2} \mathrm{Cl}(168 \mathrm{mg}, 2.06 \mathrm{mmol})$ in dry tetrahydropyran $(2.0 \mathrm{~mL})$ was added triethylamine $(0.43 \mathrm{~mL}, 3.08 \mathrm{mmol})$ under nitrogen atmosphere. The reaction mixture was allowed to stir at $80{ }^{\circ} \mathrm{C}$ for $144 \mathrm{~h}$. The resulting mixture was cooled to room temperature and quenched with $1 \mathrm{~N} \mathrm{NaOH}$ solution. The mixture was extracted with $\mathrm{CH}_{2} \mathrm{Cl}_{2}$ and the separated organic layer was 
dried over anhydrous $\mathrm{MgSO}_{4}$. After evaporation under reduced pressure, the residue was purified by flash column chromatography (hexane/ethyl acetate $=70 / 30$ ) to give 75 $\mathrm{mg}(60 \%$ yield) of 3al. Similarly, the ring-opening product 3ak ( $9 \%$ yield) was prepared under the same condition.

\section{(1S,2S)-(2-Dimethylamino-1,2-dihydro-naphthalen-1-yl)-carbamic acid tert-butyl} ester (3al): 99\% ee by HPLC analysis with a chiral column (Chiralcel OD, hexane/2-propanol =90/10, $0.5 \mathrm{~mL} / \mathrm{min}$ ), $\mathrm{t}_{\mathrm{R}}(S)-(+), 10.6 \mathrm{~min}$ (major); $(R)-(-), 13.7 \mathrm{~min}$ (minor). $[\alpha]_{\mathrm{D}}^{25}+266.0\left(c 1.00, \mathrm{CHCl}_{3}\right)$ for 3 al of $99 \%$ ee; IR $\left(\mathrm{NaCl}, \mathrm{cm}^{-1}\right) 3340,3188$, 1704, 1513, 1487, 1454, 1390, 1365, 1250, 1173, 1044, 1019. ${ }^{1} \mathrm{H}$ NMR $\left(\mathrm{CDCl}_{3}\right) \delta 1.45$ $(\mathrm{s}, 9 \mathrm{H}), 2.28(\mathrm{~s}, 6 \mathrm{H}), 3.30(\mathrm{t}, J=4.5 \mathrm{~Hz}, 1 \mathrm{H}), 4.76(\mathrm{bs}, 1 \mathrm{H}), 4.97$ (bs, 1H), $5.94(\mathrm{dd}, J=$ 9.6, 4.8 Hz, 1H), $6.63(\mathrm{~d}, J=9.6 \mathrm{~Hz}, 1 \mathrm{H}), 7.07-7.10(\mathrm{~m}, 1 \mathrm{H}), 7.21-7.23(\mathrm{~m}, 2 \mathrm{H})$, 7.32-7.34 (m, 1H); ${ }^{13} \mathrm{C} \mathrm{NMR}\left(\mathrm{CDCl}_{3}\right) \delta 155.0,135.1,132.1,129.3,128.1,127.9,127.8$, 126.6, 125.9, 79.3, 63.5, 48.1, 41.3, 28.3. HRMS (EI) calcd for $\mathrm{C}_{17} \mathrm{H}_{24} \mathrm{~N}_{2} \mathrm{O}_{2}\left(\mathrm{M}^{+}\right)$: 288.1838. Found: 288.1841.

Preparation of $(\alpha S, \alpha S)-2,2^{\prime}$-Bis $(\alpha-N, N$-dimethylaminoethyl)-(R,R)-1,1'-bis $\{$ di (4-methylphenyl)-phosphino\} ferrocene (4b): To a solution of $(S, S)-1,1^{\prime}$-bis $(\alpha-N, N$ dimethylaminoethyl)ferrocene $(1.2 \mathrm{~g}, 3.6 \mathrm{mmol})$ prepared according to the reported procedure by $\mathrm{Knochel}^{5}$ in dry ether was slowly added $n$-butyllithium solution in hexane (6.9 $\mathrm{mL}, 10.9 \mathrm{mmol}$ ) by syringe pump at room temperature for $45 \mathrm{~min}$ under nitrogen atmosphere. The reaction mixture was allowed to stir at room temperature for $2.5 \mathrm{~h}$ and di(4-methylphenyl)chlorophosphine ${ }^{12}(2.9 \mathrm{~g}, 11.7 \mathrm{mmol})$ was slowly added at room temperature. The mixture was refluxed for another $15 \mathrm{~h}$. The resulting mixture was quenched with saturated $\mathrm{NaHCO}_{3}$ solution and was extracted with benzene. The separated organic layer was dried over anhydrous $\mathrm{MgSO}_{4}$ and evaporated under reduced pressure. The residue was purified by flash column chromatography on silica gel (hexane/ethyl acetate $=50 / 50)$ to give $0.9 \mathrm{~g}\left(33 \%\right.$ yield) of $\mathbf{4 b}$. $[\alpha]^{25}{ }_{\mathrm{D}}+460.2(c 1.00$, $\left.\mathrm{CHCl}_{3}\right)$; mp 174-176 ${ }^{\circ} \mathrm{C}$; IR $\left(\mathrm{NaCl}, \mathrm{cm}^{-1}\right)$ 2966, 2929, 2812, 2796, 1596, 1496, 1447, $1271,1242,1184,1093,1019,929,804,756,623 .{ }^{1} \mathrm{H}$ NMR $\left(\mathrm{CDCl}_{3}\right) \delta 1.27(\mathrm{~d}, J=6.3$ $\mathrm{Hz}, 6 \mathrm{H}), 1.73$ (s, $12 \mathrm{H}), 2.25$ (s, 6H), 2.34 (s, 6H), 3.03 (s, 2H), 4.00-4.09 (m, 2H), 4.13 $(\mathrm{s}, 2 \mathrm{H}), 4.34(\mathrm{~s}, 2 \mathrm{H}), 6.93-6.97(\mathrm{~m}, 12 \mathrm{H}), 7.19(\mathrm{t}, J=7.7 \mathrm{~Hz}, 4 \mathrm{H}) ;{ }^{31} \mathrm{P} \mathrm{NMR}\left(\mathrm{CDCl}_{3}\right) \delta$ -25.5. Anal. Calcd for $\mathrm{C}_{46} \mathrm{H}_{54} \mathrm{FeN}_{2} \mathrm{P}_{2}$ : C, 73.40; H, 7.23; N, 3.72. Found: C, 73.53; H, $7.31 ; \mathrm{N}, 3.72$.

Preparation of (1S,2S)-2-(4-Phenyl-piperazin-1-yl)-1,2-dihydro-napthalen-1-yl -amine (5): To a stirred solution of $3 \mathbf{a c}(300 \mathrm{mg}, 0.91 \mathrm{mmol})$ in dry $\mathrm{CH}_{2} \mathrm{Cl}_{2}$, was added 
dropwise trifluoroacetic acid (dropwise addition, $1 \mathrm{~mL}$ ) at $0{ }^{\circ} \mathrm{C}$. The mixture was stirred at room temperature for $45 \mathrm{~min}$. The reaction was quenched with $10 \% \mathrm{KOH}$ and the mixture was extracted with $\mathrm{CH}_{2} \mathrm{Cl}_{2}$. The organic layer was dried over $\mathrm{Na}_{2} \mathrm{SO}_{4}$, filtered, and concentrated under reduced pressure to give $274 \mathrm{mg}$ of $\mathbf{5}$ (99\% yield) as a white solid. mp 120-123 ${ }^{\circ} \mathrm{C} ;[\alpha]^{25}$ D $+404.0\left(c 1.10, \mathrm{CHCl}_{3}\right)$; IR $\left(\mathrm{NaCl}, \mathrm{cm}^{-1}\right) 3620,3022,2400$, $1599,1522,1423,1333,1233,1046,928,720 .{ }^{1} \mathrm{H} \mathrm{NMR}\left(\mathrm{CDCl}_{3}\right) \delta 1.66(\mathrm{~s}, 2 \mathrm{H})$, 2.63-2.78 (m, 4H), 3.07-3.19 (m, 4H), 3.29-3.33 (m, 1H), $4.12(\mathrm{~d}, J=10.0 \mathrm{~Hz}, 1 \mathrm{H})$, $6.03(\mathrm{dd}, J=13.0,6.0 \mathrm{~Hz}, 1 \mathrm{H}), 6.59(\mathrm{dd}, J=13.0,2.0 \mathrm{~Hz}, 1 \mathrm{H}), 6.78-6.88(\mathrm{~m}, 3 \mathrm{H})$, 7.05-7.08 (m, 1H), $7.24(\mathrm{~m}, 4 \mathrm{H}), 7.37-7.40(\mathrm{~m}, 1 \mathrm{H}) ;{ }^{13} \mathrm{C} \mathrm{NMR}\left(\mathrm{CDCl}_{3}\right) \delta 151.1,138.9$, $131.7,129.2$, 128.9, 127.8, 127.1, 126.5, 125.7, 124.7, 119.6, 116.0, 66.9, 50.1, 49.9, 48.8. HRMS calcd for $\mathrm{C}_{20} \mathrm{H}_{23} \mathrm{~N}_{3}\left(\mathrm{M}^{+}\right)$: 305.1891. Found: 305.1899 .

\section{Preparation of (1S,2S)-4-Bromo- $N$-[2-(4-phenyl-piperazin-1-yl)-1,2-dihydro} -naphthalen-1-yl]-benzenesulfonamide (6): To a solution of 5 (300 mg, $0.98 \mathrm{mmol})$ and triethylamine $(0.27 \mathrm{~mL}, 1.96 \mathrm{mmol})$ in dry $\mathrm{CH}_{2} \mathrm{Cl}_{2}(10 \mathrm{~mL})$ was added 4-bromobenzenesulfonyl chloride (301 $\mathrm{mg}, 1.18 \mathrm{mmol}$ ) and the mixture was allowed to stir at room temperature for $16 \mathrm{~h}$. The resulting mixture was quenched with $10 \% \mathrm{KOH}$ solution and was extracted with $\mathrm{CH}_{2} \mathrm{Cl}_{2}$. After concentration under reduced pressure, the crude residue was purified by flash column chromatography (hexane/ethyl acetate $=$ $80 / 20$ ) to give $274 \mathrm{mg}$ of 6 (95\% yield); $\mathrm{mp} 188-190{ }^{\circ} \mathrm{C}$; $[\alpha]^{25}{ }_{\mathrm{D}}+224.2\left(c 0.85, \mathrm{CHCl}_{3}\right)$; IR $\left(\mathrm{NaCl}, \mathrm{cm}^{-1}\right) 3387,3014,2400,2046,1968,1912,1600,1522,1422,1331,1202$, 1045, 929, 795. ${ }^{1} \mathrm{H}$ NMR $\left(\mathrm{CDCl}_{3}\right) \delta 2.42-2.47(\mathrm{~m}, 2 \mathrm{H})$ 2.63-2.68 (m, 2H), 2.97-3.04 (m, $4 \mathrm{H}), 3.53-3.55(\mathrm{~m}, 1 \mathrm{H}), 4.62(\mathrm{~s}, 1 \mathrm{H}), 4.66-4.68(\mathrm{~m}, 1 \mathrm{H}), 5.95(\mathrm{dd}, J=9.9,6.0 \mathrm{~Hz}, 1 \mathrm{H})$, $6.69(\mathrm{~d}, J=9.9 \mathrm{~Hz}, 1 \mathrm{H}), 6.78(\mathrm{~d}, J=8.1 \mathrm{~Hz} ., 1 \mathrm{H}), 6.82-6.85(\mathrm{~m}, 3 \mathrm{H}), 7.07-7.11(\mathrm{~m}, 2 \mathrm{H})$, 7.21-7.26 (m, 4H), 7.72-7.74(m, 4H); ${ }^{13} \mathrm{C} \mathrm{NMR}\left(\mathrm{CDCl}_{3}\right) \delta 151.4,140.1,133.4,132.6$, 132.3, 130.1, 129.2, 128.9, 128.6, 127.9, 122.8, 127.8, 127.2, 124.5, 120.0, 116.4, 63.8, 51.4, 49.7, 48.6. HRMS calcd for $\mathrm{C}_{25} \mathrm{H}_{26} \mathrm{~N}_{3} \mathrm{O}_{2} \mathrm{SBr}\left(\mathrm{M}^{+}\right)$: 523.0929. Found: 523.0918.

\section{Preparation of $(1 S, 2 S)-1,2,3,4$-Tetrahydronaphthalen-1,2-diamine $(R, R)$-tartrate}

(7): To a solution of 3ae (1.0 g, $2.27 \mathrm{mmol}, 97 \%$ ee) in ethyl acetate $(15 \mathrm{~mL})$ and methanol $(30 \mathrm{~mL})$ was added ammonium formate $(1.4 \mathrm{~g}, 22.70 \mathrm{mmol})$ and $30 \mathrm{~mol} \%$ of $\mathrm{Pd} / \mathrm{C}(10 \mathrm{wt} \%, 0.7 \mathrm{~g}, 0.68 \mathrm{mmol})$. The mixture was refluxed for $25 \mathrm{~min}$ and was cooled to room temperature. The resulting black suspension was filtered through Celite and the Celite cake was washed with triethylamine/methanol $(1 / 1=\mathrm{v} / \mathrm{v})$ several times. The filtrate was concentrated under reduced pressure. The residue was treated with $50 \%$ hydrochloric acid $(80 \mathrm{~mL})$ at $100{ }^{\circ} \mathrm{C}$ for $24 \mathrm{~h}$. The reaction mixture was cooled to room temperature and the acidic solution was washed with ether several times. Then, to 
the separated aqueous solution was slowly added $4 \mathrm{~N} \mathrm{NaOH}$ solution at $0{ }^{\circ} \mathrm{C}$ for $30 \mathrm{~min}$ and its $\mathrm{pH}$ was adjusted around $\mathrm{pH} 11$. The resulting basic solution was extracted with $\mathrm{CH}_{2} \mathrm{Cl}_{2}$ and dried over anhydrous $\mathrm{MgSO}_{4}$. After removal of all volatile solvents under reduced pressure, the crude solid was dissolved with ethanol $(40 \mathrm{~mL})$ and $\mathrm{H}_{2} \mathrm{O}(5 \mathrm{~mL})$ and L-(+)-tartaric acid $(0.3 \mathrm{~g}, 1.82 \mathrm{mmol})$ solution in ethanol $(10 \mathrm{~mL})$ was slowly added. The resulting suspension was refluxed for $18 \mathrm{~h}$ and was slowly cooled to room temperature. The precipitate was collected by filtration and the collected solid was washed with cold methanol. After drying under vacuum, $0.4 \mathrm{~g}$ (52\% yield, 3 step) of $(S, S)$-diamine $(R, R)$-tartrate 7 was obtained. $[\alpha]^{25}{ }_{\mathrm{D}}+27.2\left(c 1.00, \mathrm{H}_{2} \mathrm{O}\right) ; \mathrm{mp} 238-240{ }^{\circ} \mathrm{C}$ (decomp); ${ }^{1} \mathrm{H}$ NMR $\left(d^{6}\right.$-DMSO) $\delta 1.71-1.85(\mathrm{~m}, 1 \mathrm{H}), 2.08-2.16(\mathrm{~m}, 1 \mathrm{H}), 2.80-2.84(\mathrm{~m}$, 2H), 3.02-3.10 (m, 1H), 3.80 (d, J = 9.0 Hz, 1H), 5.18 (bs, 10H), 7.09 (d, $J=7.2 \mathrm{~Hz}$, $1 \mathrm{H}), 7.15-7.24(\mathrm{~m}, 2 \mathrm{H}), 7.60(\mathrm{~d}, J=6.9 \mathrm{~Hz}, 1 \mathrm{H}) ;{ }^{13} \mathrm{C} \mathrm{NMR}\left(d^{6}\right.$-DMSO) $\delta 174.5,138.1$, 135.3, 128.1, 127.6, 126.8, 126.1, 71.4, 53.5, 53.4, 27.0, 26.4.; Anal. Calcd for $\mathrm{C}_{14} \mathrm{H}_{22} \mathrm{~N}_{2} \mathrm{O}_{7}$ : C, 50.90; H, 6.71; N, 8.48. Found: C, 51.13; H, 6.70; N, 8.44.

Rhodium-Catalyzed CO Insertion of Azabenzonorbornadiene: The mixture of 1a $(100 \mathrm{mg}, 0.41 \mathrm{mmol})$ and $\left[\mathrm{Rh}(\mathrm{CO})_{2} \mathrm{Cl}\right]_{2}(8 \mathrm{mg}, 0.02 \mathrm{mmol})$ in dry toluene $(3.0 \mathrm{~mL})$ was flushed with $\mathrm{CO}$ for 5-10 minutes and then place under a $\mathrm{CO}$ atmosphere (a balloon with $\mathrm{CO}$ is attached to the top of the reflux condenser). The mixture was allowed to stir at $110{ }^{\circ} \mathrm{C}$ for $20 \mathrm{~h}$. The solvent was removed under high vacuum and the residue was purified by flash column chromatography (hexane/ethyl acetate $=90 / 10$ ) to give $100 \mathrm{mg}$ (99\% yield) of Naphthalen-1-yl-carbamic acid tert-butyl ester (8): ${ }^{13} \mathrm{mp} 95{ }^{\circ} \mathrm{C}$; IR $\left(\mathrm{NaCl}, \mathrm{cm}^{-1}\right)$ 3329, 3051, 2978, 1694, 1531, 1494, 1392, 1367, 1345, 1234, 1157, 1102 , 1069, 996, 862. ${ }^{1} \mathrm{H}$ NMR $\left(\mathrm{CDCl}_{3}\right) \delta 1.56(\mathrm{~s}, 9 \mathrm{H}), 6.87$ (bs, 1H), 7.42-7.54 (m, 3H), 7.62 $(\mathrm{d}, J=8.4 \mathrm{~Hz}, 1 \mathrm{H}), 7.83-7.90(\mathrm{~m}, 3 \mathrm{H}) ;{ }^{13} \mathrm{C} \mathrm{NMR}\left(\mathrm{CDCl}_{3}\right) \delta 28.3,80.6,118.6,120.4$, $124.4,125.8,125.2,125.9,126.4,128.7,132.9,134.0,153.4$.

Preparation of 1-Methyl-9- $\mathrm{N}$-(4-methylbenzenesulfonyl)-1,4-dihydro-1,4epiazano-naphthalene (10): To a stirred solution of anthranilic acid (1.0 g, $7.57 \mathrm{mmol})$ and isoamylnitrite $(1.0 \mathrm{~mL}, 7.57 \mathrm{mmol})$ in dry DME $(5.0 \mathrm{~mL})$ was slowly added a solution of 2-methyl-1-(4-methylbenzenesulfonyl)- $1 H$-pyrrole ${ }^{14}(0.9 \mathrm{~g}, 3.78 \mathrm{mmol})$ in dry DME $(3.0 \mathrm{~mL})$ at $50{ }^{\circ} \mathrm{C}$ for $30 \mathrm{~min}$. The reaction was allowed to stir at $50{ }^{\circ} \mathrm{C}$ for $2 \mathrm{~h}$ until no further gas was evolved. The resulting mixture was then concentrated and purified by flash column chromatography (hexane/diethyl ether $=90 / 10$ ) to give $0.3 \mathrm{~g}$ of 10 in $27 \%$ yield. $\mathrm{mp} 165-170{ }^{\circ} \mathrm{C}$; IR $\left(\mathrm{NaCl}, \mathrm{cm}^{-1}\right) 3384,2360,1596,1453,1339,1157$, 1087, 1022, 815, 677. ${ }^{1} \mathrm{H}$ NMR $\left(\mathrm{CDCl}_{3}\right) \delta 2.03(\mathrm{~s}, 3 \mathrm{H}), 2.31(\mathrm{~s}, 3 \mathrm{H}), 5.48(\mathrm{~d}, J=2.4 \mathrm{~Hz}$, $1 \mathrm{H}), 6.47(\mathrm{dd}, J=5.5,0.9 \mathrm{~Hz}, 1 \mathrm{H}), 6.77-6.80(\mathrm{~m}, 2 \mathrm{H}), 6.81-6.85(\mathrm{~m}, 1 \mathrm{H}), 6.89-6.92(\mathrm{~m}$, 
1H), 7.02-7.05 (m, 1H), $7.06(\mathrm{~d}, J=8.1 \mathrm{~Hz}, 2 \mathrm{H}), 7.44(\mathrm{~d}, J=8.1 \mathrm{~Hz}, 2 \mathrm{H}) ;{ }^{13} \mathrm{C} \mathrm{NMR}$ $\left(\mathrm{CDCl}_{3}\right) \delta 150.2,148.1,146.6,143.0,135.9,129.2,128.3,124.9,124.9,120.9,119.2$, 75.2, 68.4, 48.2, 42.8, 21.5, 13.6. HRMS calcd for $\mathrm{C}_{18} \mathrm{H}_{17} \mathrm{NO}_{2} \mathrm{~S}\left(\mathrm{M}^{+}\right)$: 311.0980 . Found: 311.0988 .

\section{Preparation of trans-4-Methyl- $N$-[4-methyl-2-( $N$-methyl- $N$-phenylamino)-1,2-} dihydronaphthalen-1-yl]-benzenesulfonamide (11): To a solution of 10 (100 mg, $0.32 \mathrm{mmol}),[\mathrm{Rh}(\mathrm{cod}) \mathrm{Cl}]_{2}(6.0 \mathrm{mg}, 0.01 \mathrm{mmol})$, and dppf $(17.0 \mathrm{mg}, 0.03 \mathrm{mmol})$ in dry THF $(2.5 \mathrm{~mL})$ was added $N$-methylaniline $(104 \mathrm{mg}, 0.96 \mathrm{mmol})$ and the mixture was allowed to stir at $80{ }^{\circ} \mathrm{C}$ for $15 \mathrm{~h}$ under $\mathrm{N}_{2}$ atmosphere. The resulting mixture was then concentrated and purified by flash column chromatography (hexane/ethyl acetate = 70/30) to give $60 \mathrm{mg}$ of 11 (45\% yield) and $30 \mathrm{mg}$ of 12 (30\% yield) as a byproduct. mp 143-147 ${ }^{\circ} \mathrm{C}$; IR $\left(\mathrm{NaCl}, \mathrm{cm}^{-1}\right)$ 3274, 3060, 2921, 1598, 1503, 1450, 1323, 1094, 1034, 909, 813, 749, 665. ${ }^{1} \mathrm{H}$ NMR $\left(\mathrm{CDCl}_{3}\right) \delta 2.14(\mathrm{~s}, 3 \mathrm{H}) 2.30(\mathrm{~s}, 3 \mathrm{H}), 2.42$ (s, 3H), 4.50 (dd, $J=7.6,5.6 \mathrm{~Hz}, 1 \mathrm{H}), 4.61-4.65(\mathrm{~m}, 2 \mathrm{H}), 5.69$ (d, $J=4.4 \mathrm{~Hz}, 1 \mathrm{H}), 6.72-6.77(\mathrm{~m}, 2 \mathrm{H})$, $6.93(\mathrm{~d}, J=7.2 \mathrm{~Hz}, 1 \mathrm{H}), 7.09-7.14(\mathrm{~m}, 1 \mathrm{H}), 7.18-7.22(\mathrm{~m}, 2 \mathrm{H}), 7.25-7.32(\mathrm{~m}, 2 \mathrm{H}), 7.62$

$(\mathrm{d}, J=8.4 \mathrm{~Hz}, 2 \mathrm{H}) ;{ }^{13} \mathrm{C} \mathrm{NMR}\left(\mathrm{CDCl}_{3}\right) \delta 149.2,143.5,137.8,135.9,134.4,134.0,129.7$, 129.3, 128.7, 128.4, 127.9, 127.4, 123.9, 123.1, 117.5, 113.8, 58.7, 54.8, 32.4, 21.7, 19.2. HRMS calcd for $\mathrm{C}_{25} \mathrm{H}_{26} \mathrm{~N}_{2} \mathrm{O}_{2} \mathrm{~S}\left(\mathrm{M}^{+}\right)$: 418.1715. Found: 418.1710 .

N-(4-Methylbenzenesulfonyl)-4-methyl-1-amino-naphthalene (12): $\mathrm{mp}$ 152-156 ${ }^{\circ} \mathrm{C}$; IR $\left(\mathrm{NaCl}, \mathrm{cm}^{-1}\right) 3267,2924,1598,1431,1337,1161,1092,813,754 ;{ }^{1} \mathrm{H}$ NMR $\left(\mathrm{CDCl}_{3}\right) \delta 2.34(\mathrm{~s}, 3 \mathrm{H}), 2.64(\mathrm{~s}, 3 \mathrm{H}), 6.66(\mathrm{~s}, 1 \mathrm{H}), 7.15(\mathrm{~d}, J=8.0 \mathrm{~Hz}, 2 \mathrm{H}), 7.19(\mathrm{~s}, 2 \mathrm{H})$, 7.40-7.45 (m, 2H), 7.47-7.51 (m, 2H), 7.61 (dd, $J=6.8,1.6 \mathrm{~Hz}, 4 \mathrm{H}), 7.86(\mathrm{~d}, J=8.4 \mathrm{~Hz}$, $2 \mathrm{H}), 7.95(\mathrm{~d}, J=8.4 \mathrm{~Hz}, 2 \mathrm{H}) ;{ }^{13} \mathrm{C}$ NMR $\left(\mathrm{CDCl}_{3}\right) \delta 143.8,136.6,134.3,133.4,129.7$, $129.6,127.5,126.4,126.2,124.8,123.6,122.4,21.6,19.5$. HRMS calcd for $\mathrm{C}_{18} \mathrm{H}_{17} \mathrm{NO}_{2} \mathrm{~S}\left(\mathrm{M}^{+}\right)$: 311.0980 . Found: 311.0989 .

\section{References}

(1) Lautens, M.; Hiebert, S.; Renaud, J. Org. Lett. 2000, 2, 1971.

(2) Yoshikawa, K.; Bekki, K.; Karatsu, M.; Toyoda, K.; Kamio, T.; Morishima, I. J. Am. Chem. Soc. 1976, 98, 3272.

(3) (a) Lautens, M.; Fagnou, K.; Zunic, V. Org. Lett. 2002, 4, 3465. (b) Kaupp, G.; Perreten, J.; Leute, R.; Prinzbach, H. Chem. Ber. 1970, 103, 2288. 
(4) (a) Lautens, M.; Dockendorff, C. Org. Lett. 2003, 5, 3695. (b) Hodgson, D. M.; Bebbington, M. W.; Willis, P. Org. Lett. 2002, 4, 4353.

(5) Schwink, L.; Knochel, P. Chem. Eur. J. 1998, 4, 950.

(6) Carpino, L.A.; Barr, D.E. J. Org. Chem. 1966, 31, 764.

(7) (a) Davies, J.W.; Durrant, M.L.; Walker, M.P.; Belkacemi, D.; Malpass, J.R. Tetrahedron, 1992, 48, 861. (b) Priestly, W. Tetrahedron Lett. 1972, 4295.

(8) (a) Newman, M. S.; Cella, J. A. J. Org. Chem. 1973, 38, 3482. (b) Wilcox Jr., C. F.; Farley, E. J. Am. Chem. Soc. 1984, 106, 7195.

(9) Kajigaeshi, S.; Kakinami, T.; Moriwaki, M.; Tanaka, T.; Fujisaki, S.; Okamoto, T. Bull. Chem. Soc. Jpn. 1989, 62, 439.

(10) Caster, K. C.; Keck, C. G.; Walls, R. D. J. Org. Chem. 2001, 66, 2932.

(11) Ashton, P. R.; Girreser, U.; Giuffrida, D.; Kohnke, F. H.; Mathias, J. P.; Raymo, F. M.; Slawin, A. M. Z.; Stoddart, J. F.; Williams, D. J. J. Am. Chem. Soc. 1993, 115, 5422.

(12) (a) Casalnuovo, A. L.; RajanBabu, T. V.; Ayers, J. T. A.; Warrens, T. H. J. Am. Chem. Soc. 1994, 116, 9869. (b) Iwasaki, H.; Uchibori, Y.; Umeno, M. Jpn. Kokai Tokkyo Koho 1991, 5.

(13) Manna, S.; Falck, J. R.; Mioskowski, C. J. Org. Chem. 1982, 47, 5021.

(14) Lautens, M.; Fillion, E. J. Org. Chem. 1997, 62, 4418. 